\title{
INDONESIA ZERO EMISSIONS APPLICATION (EMISI): METHODOLOGY FOR CALCULATING INDIVIDUAL EMISSIONS FROM GOODS DELIVERIES, AND MARINE AND AVIATION TRANSPORT
}

MUHAMAD RIZKI, JEANLY SYAHPUTRI, NANDA NOOR, PHILLIPE GUNAWAN GAN, RETNO WIHANESTA

\section{INTRODUCTION}

Society is becoming ever more mobile, globalized, and digitalized, and there has been a significant increase in movements of passengers and goods (Noussan and Tagliapietra 2020). In 2016, transport activities accounted for more than 24 percent of global carbon dioxide $\left(\mathrm{CO}_{2}\right)$ emissions; they also contribute to the deterioration of air quality in cities (Wang and Ge 2019; Ritchie 2020; Lindau 2015). The transport sector is a major emitter of greenhouse gases (GHGs), which accelerate climate change (IPCC 2018), as well as increasing public health risks (Amalia et al. 2013). Studies report that the transportation sector accounts for approximately one-quarter of total global $\mathrm{CO}_{2}$ emissions related to energy (Ritchie 2020; Sims et al. 2015; US EPA 2021). Similarly, the Indonesian Ministry of Energy and Mineral Resources (Ministry of Energy and Mineral Resources Indonesia 2016) reported that in 2015 the transportation sector accounted for 28 percent of national GHG emissions, mostly due to fossil fuel combustion. However, because more than 50 percent (2018) of transport emissions came from aviation, marine, and road-freight transport (Ritchie 2020), more attention should be given to this sector, especially in developing countries such as Indonesia. Though the government has set up initiatives to accelerate the electrification of the transport sector, aviation, marine, and road freight are difficult to decarbonize and electrify and therefore other efforts to reduce or mitigate emissions from these sectors are important.

\section{CONTENTS}

1. Introduction ...................................................... 1

2. Emissions for Passenger Aviation and Marine Transport .......... 3

2.1 Method ........................................................... 3

2.2 Calculating Aviation Transport Emissions ..................... 4

2.3 Calculating Marine Transport Emissions ......................10

3. Emissions for Personal Logistics Transport ....................... 12

3.1 Method ..................................................... 12

3.2 Determination of Logistics Trip Chain Based on Origin and

Destination of Goods ......................................... 13

3.3 Calculating Aviation Emissions in Personal Logistics

Transport .............................................. 18

3.4 Calculating Land Emissions in Personal Logistics

Transport ................................................... 19

4. Limitations ....................................................... 26

Endnotes ......................................................... 28

Abbreviations ..................................................... 28

References ..................................................... 29

Acknowledgments.............................................. 32

About the Authors ..................................................... 32

WRI Technical Notes document methodology underpinning research publications, interactive applications, and other tools.

Suggested Citation: Rizki, M., J. Syahputri, N. Noor, P.G. Gan, R. Wihanesta. 2021. "Indonesia Zero Emissions Application (EMISI): Methodology for Calculating Individual Emissions from Goods Deliveries, and Marine and Aviation Transport." Technical Note. Jakarta, Indonesia: World Resources Institute. Available online at: https://doi.org/10.46830/ writn.20.00150 
Aviation and marine transport serve critical roles in Indonesia, given the geographical characteristics of this archipelagic country (Leung 2016). The number of passengers in the aviation and marine transport sectors almost doubled between 2009 and 2019, reaching 28.2 million air passengers and 98.5 million marine passengers (Indonesian Bureau of Statistics 2019; 2010). Furthermore, personal logistics services, meaning business-to-customer (B2C) and customerto-customer (C2C) logistics services (i.e., conventional couriers and couriers based online), have also grown in line with an increase in economic activities. This growth has further accelerated with the digitalization of Indonesia's economy and has been supported by the rise of e-commerce, where revenues increased from US $\$ 8.459$ billion in 2017 to $\$ 30.309$ billion in 2020 (Clement 2020). In fact, logistics firms that cater to e-commerce fulfillment and shipping saw up to a 40 percent increase in business in 2020, and their growth continued into the first quarter of 2021 (Jakarta Post 2021). This was triggered by the COVID-19 outbreak; with people confined to home, they increased their use of information and communication technology (ICT) to shop and order food and beverages (Abdelrhim and Elsayed 2020).

Although the number of marine and aviation passengers decreased during the pandemic, it is predicted that numbers will increase as the pandemic recedes, and lead to higher emissions. Such an increase is also predicted for personal logistics services. This is first because the Indonesian government has launched a road map for strategic urban, industrial, and tourism development from 2020 to 2024 (Ministry of National Development Planning 2020). The development, which includes infrastructure and tourist attractions, will encourage more people to travel. Second, Indonesia is continuously improving the accessibility and quality of its ICT infrastructure. It aims not only to promote the digital economy, online activities, and tourism, but also to facilitate e-logistics services (i.e., delivery of food and beverages, and e-commerce) (Das et al. 2016; Roberts et al. 2019). Last, the Indonesian government also disburses social and financial incentives to the public, and to small and large businesses as a response to COVID-19 (Bhwana 2020). This policy aims to increase consumption and boost the economy, which in turn leads to increased mobility (Ministry of National Development Planning 2020).

While marine, aviation, and logistics services are expected to increase in the next decades and contribute to climate change, Indonesians, however, still tend to deny that their activities contribute to this issue. In fact, a study by YouGov in 2019 found that 18 percent of Indonesia's population still argue that human activity does not contribute to the climate crisis (Hilman and Harvey 2019). Given the climate emergency, it is important to establish locally relevant and practical platforms that can educate Indonesians about emissions from their aviation and marine transport, and from logistics activities.

The Indonesia Zero Emissions Application (EMISI) has since 2020 enabled Indonesians to learn, measure, track, and act on their climate impacts by helping them to calculate, reduce, and sequestrate their own personal emissions. A previous study (Rizki et al. 2020) provided methodologies for calculating emissions and pollutants for Indonesia's urban transport sector, meaning car, motorcycle, train, bus, paratransit, for the EMISI app. This technical note describes the methodologies the next iteration of EMISI will use for calculating emissions from personal aviation and marine transport, and from logistics. Personal aviation and marine transport refers to passengers using aviation transport (i.e., airplanes) and marine transport (i.e., ferries, including ro-ro ${ }^{1}$ ), while personal logistics transport refers to the domestic delivery of personal goods, both $\mathrm{B} 2 \mathrm{C}$ and $\mathrm{C}_{2} \mathrm{C}$. The $\mathrm{B} 2 \mathrm{C}$ delivery refers to the final phase of delivery, when the product is sent directly from seller to end customer, without any individual or organization, such as a supplier or distributor, in between. By including these calculations, EMISI aims to educate people about the impact of their transport behavior and their personal logistics activities (e.g., e-shopping and sending packages) on climate change and provide them with opportunities to mitigate this.

This technical note focuses on calculating methane $\left(\mathrm{CH}_{4}\right)$ and $\mathrm{CO}_{2}$ as GHG emissions, followed by carbon monoxide (CO), nitrogen oxides $\left(\mathrm{NO}_{\mathrm{x}}\right)$, fine particulate matter $\left(\mathrm{PM}_{2.5}\right.$, meaning particulate matter with diameter less than 2.5 micrometers), sulfur dioxide $\left(\mathrm{SO}_{2}\right)$, and nonmethane volatile organic compounds (NMVOCs) as air pollutants, considering their substantial implications for climate change and air pollution. Consequently, further adjustments to methodologies of the Intergovernmental Panel on Climate Change (IPCC) are made by adopting Indonesia-specific emission factors, coefficients, and assumptions from best available government data (Ministry of Energy and Mineral Resource Indonesia 2017; Ministry of Environment and Forestry Indonesia 2017; 2010), with complementary international sources (ICAO 2016; Olmer et al. 2017; United Nations 2020). 


\section{EMISSIONS FOR PASSENGER AVIATION AND MARINE TRANSPORT}

\subsection{Method}

The Guidelines for National Greenhouse Gas Inventories (Eggleston et al. 2006) classifies transport activities as emissions from mobile sources. According to the guidelines, GHG and air pollutant emissions can be calculated using a top-down or bottom-up approach. A top-down approach uses aggregate data, such as the total fuel consumed in a country, while the bottom-up approach calculates the emissions or air pollutants from data relating to individual trips. The methodology to calculate aviation and marine transport emissions uses a bottom-up approach; EMISI gathers data on the travel of individuals.
This section describes the methodology for calculating emissions from aviation and marine passenger transport, while the next focuses on aviation and marine logistics transport. Figure 1 displays the calculation framework for aviation and marine passenger transport, where several parameters are needed to calculate emissions and air pollutants. The gray boxes represent the process of calculating, the green boxes represent the calculation output, and the boxes in other colors represent secondary data. As the bottom-up approach requires activity data from individuals, users input their airport or seaport of origin and destination. This is used to generate an individual's travel distance using the Google Maps app, which is included in EMISI.

Figure 1 | Aviation and Marine Passenger Transport Method Framework

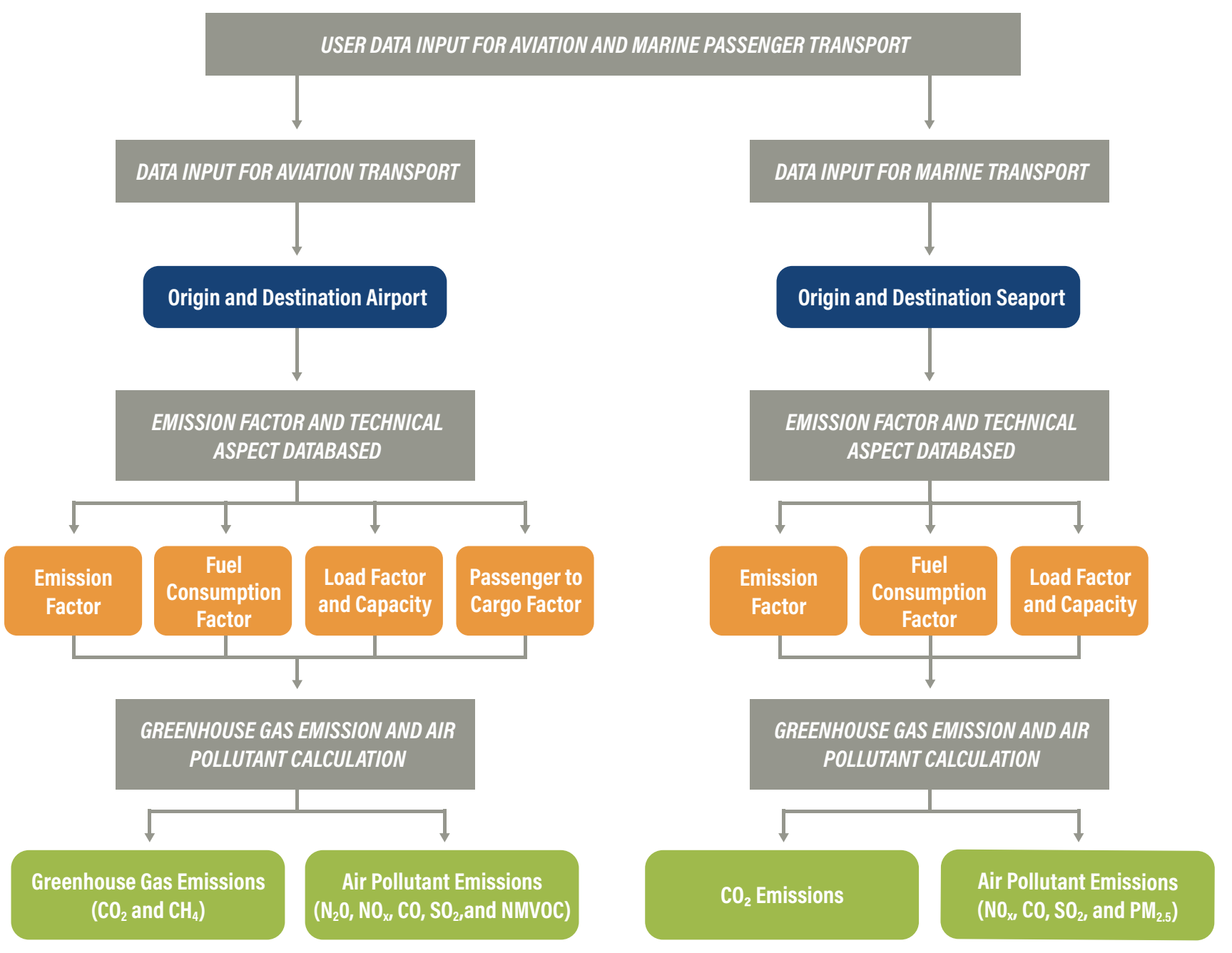

Note: Blue boxes indicate data input, orange: based on empirical data; and green indicate output. Sources: WRI Indonesia 
The emissions and air pollutants caused by individuals are calculated based on two types of considerations. The first are technical determinants related to an aircraft or ship, such as capacity, load factor, passenger-tocargo factor, and fuel consumption factor. For aviation transport, EMISI uses data from various publications and reports, mainly from Indonesian airlines (i.e., Garuda Indonesia, Lion, etc.) and the International Civil Aviation Organization (ICAO 2018). For marine transport, the methodology only focuses on ferry or ro-ro services, which are commonly used as passenger vessels (Utami 2020). Technical data is gathered from publications and reports from the Ministry of Transportation (Ministry of Transportation 2014). Second, emission-related factors such as emission factors for $\mathrm{CO}_{2}, \mathrm{SO}_{2}$, and $\mathrm{NOx}$ pollutants must be considered. The EMISI app uses emission factors from the ICAO (2018), IPCC (2006), and local conversion factors from the Ministry of Energy and Mineral Resource (2017).

\subsection{Calculating Aviation Transport Emissions}

\section{Method: Calculating Greenhouse Gas and Air Pollutant Emissions}

Emissions of GHGs and air pollutants of passenger aviation are calculated according to the ICAO (2018) and Ministry of Energy and Mineral Resources (2017). To simplify the information required from users, the ICAO designed a fuel-based approach using aircraft-type data and factors (i.e., fuel consumption, correction) related to distance, and route data. Under a fuel-based approach, emissions calculations are based on the fuel consumption of every trip per individual. The fuel consumption calculation consists of fuel consumed during the landing and take-off period (LTO), including taxiing, and climbcruise-descent time (CCD). As mentioned in ICAO (2020), the LTO period specifically refers to activity below 914 $\mathrm{m}$ (3,00o feet). To convert from trip-based calculation to individual-based, the formula also considers technical factors such as the capacity and load factor of the aircraft. For the calculation, users are required to enter the route of their trip and the type of aircraft. If journeys are indirect, individuals must calculate the GHG emissions and air pollution for each leg of their journey.

The formula for the fuel-based method to calculate GHG emissions and air pollutants is as follows:

Total aviation emissions per person $\left(T A E P_{p e}\right)$

$=\sum_{i=1}^{N}\left(\left(\left(E F_{C C D \text { e } / \text { ap }} \times D c_{i} x F \mathrm{a}_{C C D-\mathrm{j}}\right)+E F_{L T O e / a p}\right) \times \frac{P C F_{i}}{S_{i j} \times P L F_{i}}\right)$

where $i(\mathrm{i}=1,2, \ldots \mathrm{N})$ is the number of trips $\left(\mathrm{i}^{\text {th }}\right) ; j$ is the aircraft type; $E F_{C C D e / a p}$ is emission/pollutant (e/ap) factor ( $\mathrm{kg}(\mathrm{GHG} / \mathrm{p}) / \mathrm{kg}$ fuel) during CCD; $D c_{i}$ is the total distance in the $i^{\text {th }}$ trip (kilometers; $\mathrm{km}$ ), which is corrected with a distance correction factor; $F a_{C C D-j}$ is fuel consumption factor $(\mathrm{kg}$ fuel $/ \mathrm{km})$ of $j$ - aircraft during cruise time; $E F_{\text {Lто }}$ e/ap is emission/pollutant (e/ap) factor ( $\mathrm{kg}(\mathrm{e}) / \mathrm{LTO}$ cycle) during LTO; $P C F_{i}$ is passenger-to-cargo factor or the ratio of number of passengers to tonnage of mail and freight for the $\mathrm{i}^{\text {th }}$ trip; $S_{i j}$ is the total number of seats available based on the aircraft type $(j)$ for the $i^{t h}$ trip; and $P L F_{i}$ is passenger load factor or the ratio of number of passengers transported and the number of available seats for the $\mathrm{i}^{\text {th }}$ trip. The total emissions and air pollutants per person is the sum of the calculation for every stage of the journey.

\section{Technical Determinant for Calculating Greenhouse Gas and Air Pollutant Emissions}

The technical determinants for calculating aviation emissions are aircraft-related characteristics and parameters including fuel consumption, load factor, number of seats, and passenger-to-cargo factor. A correction factor-shown in Table 1-should be added to the trip distance when determining the fuel consumption during CCD (see Table 2). This correction factor represents excess distance including stacking, waiting, and weatherdriven correction (ICAO 2018) and is essential to correctly calculate GHG emissions and air pollutants per person.

Table 1 | Distance Correction

\begin{tabular}{|l|l|}
\hline Trip Distance $(\mathrm{km})$ & Distance Correction Factor $(\mathrm{km})$ \\
\hline Less than 550 & 50 \\
\hline Between $550 \mathrm{~km}$ and 5500 & 100 \\
More than 5500 & 125 \\
\hline
\end{tabular}

Source: ICA0. 2018. "Airport Air Quality Manual. Doc 9889." www.icao.int/environmental-protection/ CarbonOffset/Documents/Methodology\%20ICA0\%20Carbon\%20Calculator_v11-2018.pdf.

The fuel consumption factor during the CCD period is described in Table 2. As the CCD period substantially depends on the distance traveled, the fuel consumption factor will be based on distance, the unit for which is kilograms of fuel per kilometer. The ICAO (2018) assesses fuel consumption based on type of engine and distance, and the resulting number is for the entire flight including LTO and CCD. Therefore, to calculate the CCD fuel consumption according to ICAO 2018, the fuel consumption during LTO has to be subtracted from the total fuel consumption. For both LTO and CCD, the fuel consumption factors employed are for those engines commonly used by Indonesian aircraft. Furthermore, while ICAO (2018) also divides the fuel emission factors based on distance (e.g., 150 nautical miles (nm), $250 \mathrm{~nm}, 500 \mathrm{~nm}, 750 \mathrm{~nm}, 1000 \mathrm{~nm}$, etc.), this technical note limits the distance to $2000 \mathrm{~nm}$ for domestic flights and $3500 \mathrm{~nm}$ for international flights, considering the capacity of local aircraft. Fuel consumption factors are calculated by dividing fuel consumption by distance. Results can be seen in Table 2. 
Table 2 | Fuel Consumption during Climb-Cruise-Descent Period

\begin{tabular}{|c|c|c|c|c|c|c|c|c|c|c|}
\hline \multirow{3}{*}{$\begin{array}{l}\text { Aircraft } \\
\text { Maker }\end{array}$} & \multirow{3}{*}{ Aircraft } & \multicolumn{8}{|c|}{ Total Climb-Cruise-Descent Fuel Consumption for Varying Flight Distances (kg fuel/km) } & \multirow{3}{*}{$\begin{array}{c}\text { Average } \\
\text { (kg fuel } / \mathrm{km})\end{array}$} \\
\hline & & $125 \mathrm{~nm}$ & $250 \mathrm{~nm}$ & $500 \mathrm{~nm}$ & $750 \mathrm{~nm}$ & $1000 \mathrm{~nm}$ & $1500 \mathrm{~nm}$ & $2000 \mathrm{~nm}$ & $3500 \mathrm{~nm}$ & \\
\hline & & $\sim 231 \mathrm{~km}$ & $\sim 463 \mathrm{~km}$ & $\sim 926 \mathrm{~km}$ & $\sim 1389 \mathrm{~km}$ & $\sim 1852 \mathrm{~km}$ & $\sim 2778 \mathrm{~km}$ & $\sim 3704 \mathrm{~km}$ & $\sim 4630 \mathrm{~km}$ & \\
\hline \multirow{12}{*}{ Airbus } & A320-200CE0 [m] & 3.90 & 5.75 & 4.12 & 3.92 & 3.78 & 3.60 & 3.48 & 3.48 & 4.00 \\
\hline & A320-200NEO [m] & 3.90 & 5.75 & 4.12 & 3.92 & 3.78 & 3.60 & 3.48 & 3.48 & 4.00 \\
\hline & A320-200 & 3.90 & 5.75 & 4.12 & 3.92 & 3.78 & 3.60 & 3.48 & 3.48 & 4.00 \\
\hline & A320NEO & 3.90 & 5.75 & 4.12 & 3.92 & 3.78 & 3.60 & 3.48 & 3.48 & 4.00 \\
\hline & A321NE0 & 3.08 & 5.33 & 3.91 & 3.78 & 3.68 & 3.53 & 3.43 & 3.43 & 3.77 \\
\hline & A330-200 [m] & 5.47 & 10.90 & 8.37 & 8.17 & 8.00 & 7.75 & 7.57 & 7.23 & 7.93 \\
\hline & A330-300 & 5.47 & 10.90 & 8.37 & 8.17 & 8.00 & 7.75 & 7.57 & 7.23 & 7.93 \\
\hline & A330-300[m] & 5.47 & 10.90 & 8.37 & 8.17 & 8.00 & 7.75 & 7.57 & 7.23 & 7.93 \\
\hline & A330-900NEO [m] & 5.47 & 10.90 & 8.37 & 8.17 & 8.00 & 7.75 & 7.57 & 7.23 & 7.93 \\
\hline & A330-900NE0 & 5.47 & 10.90 & 8.37 & 8.17 & 8.00 & 7.75 & 7.57 & 7.23 & 7.93 \\
\hline & Average & 4.60 & 8.28 & 6.22 & 6.03 & 5.88 & 5.67 & 5.52 & 5.35 & 5.94 \\
\hline & Average [m] & 4.60 & 8.28 & 6.22 & 6.03 & 5.88 & 5.67 & 5.52 & 5.35 & 5.94 \\
\hline \multirow{14}{*}{ Boeing } & $737 \operatorname{Max} 8$ & 1.88 & 4.71 & 3.52 & 3.45 & 3.38 & 3.26 & 3.16 & 2.97 & 3.29 \\
\hline & $737 \operatorname{Max} 8$ [m] & 1.88 & 4.71 & 3.52 & 3.45 & 3.38 & 3.26 & 3.16 & 2.97 & 3.29 \\
\hline & $737-300$ & 3.95 & 5.74 & 4.03 & 3.80 & 3.64 & 3.43 & 3.29 & 3.04 & 3.87 \\
\hline & $737-300[\mathrm{~m}]$ & 3.95 & 5.74 & 4.03 & 3.80 & 3.64 & 3.43 & 3.29 & 3.04 & 3.87 \\
\hline & $737-500$ & 3.95 & 5.74 & 4.03 & 3.80 & 3.64 & 3.43 & 3.29 & 3.04 & 3.87 \\
\hline & $737-500[\mathrm{~m}]$ & 3.95 & 5.74 & 4.03 & 3.80 & 3.64 & 3.43 & 3.29 & 3.04 & 3.87 \\
\hline & $737-800$ & 3.52 & 5.53 & 3.93 & 3.72 & 3.58 & 3.39 & 3.26 & 3.03 & 3.75 \\
\hline & $737-800 \mathrm{NG}[\mathrm{m}]$ & 3.52 & 5.53 & 3.93 & 3.72 & 3.58 & 3.39 & 3.26 & 3.03 & 3.75 \\
\hline & 737-900ER & 3.52 & 5.53 & 3.93 & 3.72 & 3.58 & 3.39 & 3.26 & 3.03 & 3.75 \\
\hline & $737-900$ ER $[\mathrm{m}]$ & 3.52 & 5.53 & 3.93 & 3.72 & 3.58 & 3.39 & 3.26 & 3.03 & 3.75 \\
\hline & $777-300$ ER & 4.89 & 11.36 & 8.98 & 8.91 & 8.80 & 8.62 & 8.48 & 8.20 & 8.53 \\
\hline & $777-300$ ER [m] & 4.89 & 11.36 & 8.98 & 8.91 & 8.80 & 8.62 & 8.48 & 8.20 & 8.53 \\
\hline & Average & 3.62 & 6.43 & 4.74 & 4.57 & 4.44 & 4.25 & 4.12 & 3.89 & 4.51 \\
\hline & Average [m] & 3.62 & 6.43 & 4.74 & 4.57 & 4.44 & 4.25 & 4.12 & 3.89 & 4.51 \\
\hline \multirow{3}{*}{ Other } & ATR72-600 & 0.92 & 1.39 & 1.21 & 1.19 & 1.18 & 1.19 & 1.22 & 1.22 & 1.19 \\
\hline & CRJ1000 & 2.45 & 3.18 & 2.23 & 2.03 & 1.92 & 1.79 & 1.79 & 1.79 & 2.15 \\
\hline & Average & 1.69 & 2.29 & 1.72 & 1.61 & 1.55 & 1.49 & 1.50 & 1.50 & 1.67 \\
\hline
\end{tabular}

Note: 1 nautical mile $(\mathrm{nm})=1.852$ kilometers $(\mathrm{km}) ;[\mathrm{m}]$ means multiple classes of seating are available.

Source: ICA0. 2018. "Airport Air Quality Manual. Doc 9889." www.icao.int/environmental-protection/CarbonOffset/Documents/Methodology\%20ICA0\%20Carbon\%20Calculator_v11-2018.pdf. 
The passenger-to-cargo factor expresses the contribution of passenger mass to total mass. Total mass consists of passengers, seats, freight/cargo, and mail (ICAO 2018). The passenger-to-cargo factor, as shown in Table 3, is divided based on travel route. This technical note compiled the PCF data from ICAO (2017) and CEIC (2020). However, the routes shown are limited to travel within Indonesia and travel with Indonesia as either origin or destination.

\section{Table 3 | Passenger-to-Cargo Factor}

\begin{tabular}{|l|l|}
\hline Route & $\begin{array}{l}\text { Passenger-to-Cargo } \\
\text { Factor (\%) }\end{array}$ \\
\hline Africa-Indonesia $^{a}$ & 83.90 \\
\hline Central Asia and Southwest Asia-Indonesia $^{\text {a }}$ & 80.65 \\
\hline Europe-Indonesia $^{a}$ & 63.49 \\
\hline Intra Indonesia $^{b}$ & 62.09 \\
\hline Intra Pacific Southeast Asia-Indonesia $^{\text {a }}$ & 79.99 \\
\hline Middle East-Indonesia $^{\text {a }}$ & 81.26 \\
\hline North America-Indonesia $^{\text {a }}$ & 84.44 \\
\hline North Asia-Indonesia & \\
\hline
\end{tabular}

Sources: ${ }^{\text {a ICAO } .2018 . ~ " A i r p o r t ~ A i r ~ Q u a l i t y ~ M a n u a l . ~ D o c ~ 9889 . " ~ w w w . i c a o . i n t / e n v i r o n m e n t a l-~}$ protection/CarbonOffset/Documents/Methodology\%20ICA0\%20Carbon\%20Calculator_v112018.pdf.; b CEIC. 2020. Indonesia Airline Production. CEIC. www.ceicdata.com/en/indonesia/ airline-production.

Seating capacity/passenger capacity is used to calculate the average number of passengers in the aircraft; seating capacity is multiplied by the passenger load factor. There are many airlines operating in Indonesia; this technical note describes seating capacity for Garuda Indonesia and Lion Air, the largest. Seating capacity, also categorized by aircraft type, is illustrated in Table 4 .
Table 4 | Average Aircraft Seating Capacity

\begin{tabular}{|c|c|c|}
\hline Aircraft & Code & Average Seating Capacity \\
\hline \multirow[t]{12}{*}{ Airbus } & 320-200CE0 [m] ${ }^{c}$ & 156 \\
\hline & 320-200NEO [m] ${ }^{c}$ & 156 \\
\hline & A320-200 d & 180 \\
\hline & A320NEO ${ }^{d}$ & 180 \\
\hline & A321NEO $^{d}$ & 236 \\
\hline & A330-200 [m] ${ }^{\mathrm{a}}$ & 222 \\
\hline & $\mathrm{A} 330-300^{\mathrm{b}}$ & 440 \\
\hline & $\mathrm{A} 330-300[\mathrm{~m}]^{\mathrm{a}}$ & 251 \\
\hline & A330-900NEO $[\mathrm{m}]^{\mathrm{a}}$ & 301 \\
\hline & A330-900NEOd & 365 \\
\hline & Average & 280 \\
\hline & Average $[\mathrm{m}]$ & 217 \\
\hline \multirow[t]{14}{*}{ Boeing } & 737 Max $8^{b}$ & 108 \\
\hline & $737 \operatorname{Max} 8[\mathrm{~m}]^{\mathrm{a}}$ & 170 \\
\hline & $737-300^{d}$ & 149 \\
\hline & $737-300[\mathrm{~m}]^{\mathrm{d}}$ & 126 \\
\hline & $737-500^{d}$ & 138 \\
\hline & $737-500^{d}[\mathrm{~m}]$ & 110 \\
\hline & $737-800^{b}$ & 189 \\
\hline & 737-800NG [m] ${ }^{\mathrm{a}}$ & 162 \\
\hline & $737-900 \mathrm{ER}^{\mathrm{b}}$ & 213 \\
\hline & $737-900 E R[m]^{c}$ & 190 \\
\hline & 777-300ER & 396 \\
\hline & $777-300 \mathrm{ER}[\mathrm{m}]^{\mathrm{a}}$ & 314 \\
\hline & Average & 198 \\
\hline & Average [m] & 178 \\
\hline \multirow[t]{3}{*}{ Other } & ATR72-600 & 70 \\
\hline & CRJ1000 & 96 \\
\hline & Average & 83 \\
\hline
\end{tabular}

Note: [m] means multiple classes of seating are available; seating capacity excludes cockpit and cabin crew(s).

Sources: a Garuda Indonesia. 2020. "Revitalisasi Armada." 2020. www.garuda-indonesia.com/ id/id/garuda-indonesia-experience/fleets/fleet-revitalization; ' Lion Air. 2020. "Armada Kami." www.lionair.co.id/tentang-kami/armada-kami; c Husaini, Azis. 2020. "Batik Air, maskapai pesaing Garuda yang kini telah operasikan 76 pesawat." kontan.co.id. May 9. http://industri. kontan.co.id/news/batik-air-maskapai-pesaing-garuda-yang-kini-telah-operasikan-76-pesawat ${ }^{d}$ Casanova, Albert M., Bert van Leeuwen, Coen Capelle, Simon Finn, and Steven Guo, 2017, "An Overview of Commercial Aircraft 2018-2019." Schipol/London: DVB Bank. www.dvbbank.com/ / media/Files/D/dvbbank-corp/aviation/dvb-overview-of-commercial-aircraft-2018-2019.pdf. 
The PLF expresses the average number of passengers in the aircraft divided by its capacity. The PLF of domestic flights on multiple Indonesian airlines is provided by the Ministry of Transportation (2018). For international flights, PLFs are provided by the ICAO (2017) according to the trip route. The domestic and international flight PLFs are summarized in Table 5.

\section{Table 5 | Passenger Load Factors for Domestic and International Flights}

\begin{tabular}{|c|c|}
\hline \multicolumn{2}{|l|}{ Domestic Flights } \\
\hline Airline & $\begin{array}{l}\text { PLF/ } \\
\text { Passenger } \\
\text { Load Factor }\end{array}$ \\
\hline Air Asia ${ }^{a}$ & 79 \\
\hline Batik Air ${ }^{a}$ & 75 \\
\hline Citilink $^{a}$ & 84 \\
\hline Garuda Indonesia $^{a}$ & 74 \\
\hline Lion Air ${ }^{a}$ & 81 \\
\hline Malindo Air ${ }^{b}$ & 76 \\
\hline Nam Air & 79 \\
\hline Sriwijaya Air & 82 \\
\hline Wings Air ${ }^{\mathrm{a}}$ & 70 \\
\hline Other ${ }^{b}$ & 78 \\
\hline \multicolumn{2}{|l|}{ International Flights } \\
\hline Airline & $\begin{array}{l}\text { Passenger } \\
\text { Load Factor } \\
(\%)\end{array}$ \\
\hline Air Asia ${ }^{a}$ & 76 \\
\hline Batik Air ${ }^{\mathrm{a}}$ & 61 \\
\hline Citilink $^{a}$ & 71 \\
\hline Garuda Indonesia $^{a}$ & 71 \\
\hline Lion Aira & 81 \\
\hline Nam Air ${ }^{\mathrm{a}}$ & 65 \\
\hline Sriwijaya Air ${ }^{\mathrm{a}}$ & 85 \\
\hline Wings Air ${ }^{\mathrm{a}}$ & 63 \\
\hline Other domestic airlines ${ }^{a}$ & 74 \\
\hline International airlines (origin/destination: Africac) & 73 \\
\hline $\begin{array}{l}\text { International airlines (origin/destination: Central Asia } \\
\text { and Southwest } A \operatorname{sia}^{c} \text { ) }\end{array}$ & 77 \\
\hline International airlines (origin/destination: Europec) & 80 \\
\hline $\begin{array}{l}\text { International airlines (origin/destination: Intra Pacific } \\
\text { Southeast Asiac) }\end{array}$ & 76 \\
\hline International airlines (origin/destination: Middle Eastc) & 78 \\
\hline $\begin{array}{l}\text { International airlines (origin/destination: North } \\
\text { Americac }^{c} \text { ) }\end{array}$ & 78 \\
\hline International airlines (origin/destination: North $\mathrm{Asia}^{\mathrm{c}}$ ) & 78 \\
\hline
\end{tabular}

Sources: a Ministry of Transportation. 2018. "Transportation Statistics 2018." http://dephub.go.id/

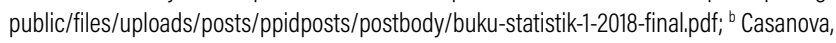
Albert M., Bert van Leeuwen, Coen Capelle, Simon Finn, and Steven Guo. 2017. "An Overview of Commercial Aircraft 2018-2019." Schipol/London: DVB Bank. www.dvbbank.com/ / media/Files/D/dvbbank-corp/aviation/dvb-overview-of-commercial-aircraft-2018-2019.pdf; c ICAO. 2018. "Airport Air Quality Manual. Doc 9889." www.icao.int/environmental-protection/ CarbonOffset/Documents/Methodology\%20ICA0\%20Carbon\%20Calculator_v11-2018.pdf.

\section{Determinants of Emissions of Greenhouse Gases and Air Pollutants}

The bottom-up approach uses emission factors to calculate emissions of GHGs - $\mathrm{CO}_{2}$ and $\mathrm{CH}_{4}$ - and air pollutants - $\mathrm{CO}$, nitrous oxide $\left(\mathrm{N}_{2} \mathrm{O}\right), \mathrm{NO}_{\mathrm{X}}$ NMVOCs, and $\mathrm{SO}_{2}$. These factors express the calculated ratio between the amount of GHG and air pollutant emissions per unit of fuel consumption. The emission factors for $\mathrm{CO}_{2}$ are based on fossil fuel consumption. As suggested by Indonesia's Ministry of Environment and Forestry (2010), emission factors are adopted from the IPCC's Guidelines for National Greenhouse Gas Inventories (Eggleston et al. 2006), which measured a default emission factor of 70,000 tonnes per terajoule (TJ) released by kerosene and aviation gasoline. In particular, the fossil fuel emission factors resulted from conversions: Indonesian emission factors from the Ministry of Environment and Forestry (2017) and the Ministry of Energy and Mineral Resources (2017) in kilograms of $\mathrm{CO}_{2}$ per TJ are multiplied by the heating value of the fuel (TJ/liter).

Calculating passenger emissions of air pollutants ( $\mathrm{CO}$, $\mathrm{N}_{2} \mathrm{O}, \mathrm{NO}_{\mathrm{X}}, \mathrm{NMVOCs}$, and $\mathrm{SO}_{2}$ ) in kilograms requires emission factors for each air pollutant. The required data is similar to that needed for the GHG emissions calculation. Table 6 and Table 7 present emission factors of LTO and CCD, respectively, sourced from the ICAO (2020) and Eggleston et al. (2006), as suggested by Indonesia's Ministry of Environment (2012). In addition, the emission factors from the IPCC (Eggleston et al. 2006) were initially obtained in tonnes per TJ, and subsequently converted into unitless emission factors with conversion factors suggested by the UN (2020). 
Table 6 | Landing-Take-Off: Emission Factors for GHGs and Air Pollutants

\begin{tabular}{|c|c|c|c|c|c|c|c|c|}
\hline \multirow{2}{*}{ Aircraft } & \multirow{2}{*}{ Code } & & \multicolumn{6}{|c|}{ Landing-Take-0ff Emission Factor (kg/Landing-Take-0ff) } \\
\hline & & $\mathrm{CO}_{2}^{\mathrm{a}}$ & $\mathrm{CH}_{4}{ }^{\mathrm{b}}$ & $\mathrm{CO}^{\mathrm{a}}$ & $\mathrm{N}_{2} \mathrm{O}^{\mathrm{a}}$ & NOx & NMVOC $^{b}$ & $\mathrm{SO}_{2}^{\mathrm{a}}$ \\
\hline \multirow{12}{*}{ Airbus } & A320-200CEO [m] & 2665 & 0.06 & 8.14 & 0.10 & 9.90 & 0.51 & 0.42 \\
\hline & A320-200NE0 [m] & 1981 & 0.06 & 6.95 & 0.10 & 5.95 & 0.51 & 0.31 \\
\hline & A320-200 & 2665 & 0.06 & 8.14 & 0.10 & 9.90 & 0.51 & 0.42 \\
\hline & A320NE0 & 1981 & 0.06 & 6.95 & 0.10 & 5.95 & 0.51 & 0.31 \\
\hline & A321NE0 & 2373 & 0.14 & 6.94 & 0.10 & 10.76 & 1.27 & 0.38 \\
\hline & A330-200 [m] & 7052 & 0.13 & 16.20 & 0.20 & 35.57 & 1.15 & 1.12 \\
\hline & A330-300 & 7052 & 0.13 & 16.20 & 0.20 & 35.57 & 1.15 & 1.12 \\
\hline & A330-300[m] & 7052 & 0.13 & 16.20 & 0.20 & 35.57 & 1.15 & 1.12 \\
\hline & A330-900NEO [m] & 7052 & 0.13 & 16.20 & 0.20 & 35.57 & 1.15 & 1.12 \\
\hline & A330-900NEO & 7052 & 0.13 & 16.20 & 0.20 & 35.57 & 1.15 & 1.12 \\
\hline & Average & 4693 & 0.10 & 11.81 & 0.15 & 22.03 & 0.91 & 0.37 \\
\hline & Average $[\mathrm{m}]$ & 4693 & 0.10 & 11.81 & 0.15 & 22.03 & 0.91 & 0.37 \\
\hline \multirow{14}{*}{ Boeing } & 737 Max 8 & 2784 & 0.07 & 7.07 & 0.10 & 6.98 & 0.65 & 0.44 \\
\hline & $737 \operatorname{Max} 8[\mathrm{~m}]$ & 2784 & 0.07 & 7.07 & 0.10 & 6.98 & 0.65 & 0.44 \\
\hline & $737-300$ & 2737 & 0.08 & 6.48 & 0.10 & 6.98 & 0.75 & 0.43 \\
\hline & $737-300[\mathrm{~m}]$ & 2737 & 0.08 & 6.48 & 0.10 & 6.98 & 0.75 & 0.43 \\
\hline & $737-500$ & 2737 & 0.08 & 6.48 & 0.10 & 6.98 & 0.75 & 0.43 \\
\hline & $737-500[\mathrm{~m}]$ & 2737 & 0.08 & 6.48 & 0.10 & 6.98 & 0.75 & 0.43 \\
\hline & $737-800$ & 2784 & 0.07 & 7.07 & 0.10 & 12.30 & 0.65 & 0.44 \\
\hline & 737-800NG [m] & 2784 & 0.07 & 7.07 & 0.10 & 12.30 & 0.65 & 0.44 \\
\hline & $737-900$ ER & 2784 & 0.07 & 7.07 & 0.10 & 12.30 & 0.65 & 0.44 \\
\hline & $737-900$ ER [m] & 2784 & 0.07 & 7.07 & 0.10 & 12.30 & 0.65 & 0.44 \\
\hline & 777-300ER & 7197 & 0.07 & 16.60 & 0.30 & 37,47 & 0.59 & 1.14 \\
\hline & $777-300$ ER [m] & 7197 & 0.07 & 16.60 & 0.30 & 37.47 & 0.59 & 1.14 \\
\hline & Average & 3520 & 0.07 & 8.46 & 0.13 & 13.84 & 0.67 & 0.55 \\
\hline & Average [m] & 3520 & 0.07 & 8.46 & 0.13 & 13.84 & 0.67 & 0.55 \\
\hline \multirow{3}{*}{ Other } & ATR72-600 & 641 & 0.30 & 2.35 & 1.88 & 10.20 & 2.60 & 0.10 \\
\hline & CRJ1000 & 1517 & 0.30 & 4.12 & 2.27 & 10.20 & 2.60 & 0.24 \\
\hline & Average & 849 & 0.30 & 3.24 & 2.08 & 10.20 & 2.60 & 0.17 \\
\hline
\end{tabular}

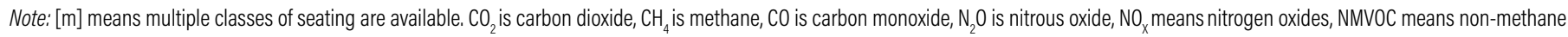
volatile organic compound, and $\mathrm{SO}_{2}$ is sulfur dioxide.

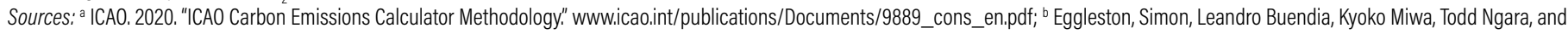
Kyoto Tanabe. 2006. 2006 IPCC Guidelines for National Greenhouse Gas Inventories. IPCC. www.ipcc-nggip.iges.or.jp/public/2006gl/.

Table 7 | Climb-Cruise-Descent: Emission Factors for Greenhouse Gas and Air Pollutant Emissions

\begin{tabular}{l|l|l|l|l|l|l|l}
\hline \multirow{2}{*}{ Flight Type } & \multicolumn{7}{|c|}{ Climb-Cruise-Descent Emission Factor (kg/kg fuel) } \\
\cline { 2 - 9 } & $\mathbf{C O}_{\mathbf{2}}$ & $\mathbf{C H}_{\mathbf{4}}$ & $\mathbf{C O}$ & $\mathbf{N}_{\mathbf{2}} \mathbf{0}$ & $\mathbf{N O}_{\mathbf{x}}$ & $\mathbf{N M V O}$ & $\mathbf{S O}_{\mathbf{2}}$ \\
\hline Domestic & 3.15 & 0.02 & 7.0 & 0.001 & 0.011 & 0.0007 & 1.0 \\
International & 3.15 & 0.02 & 5.0 & 0.001 & 0.017 & 0.0027 & 1.0 \\
\hline
\end{tabular}

Note: Domestic and international aviation emission factors are derived from emission factors of typical aircraft for domestic and international flights as suggested by Eggleston et al. (2006). $\mathrm{CO}_{2}$ is carbon dioxide, $\mathrm{CH}_{4}$ is methane, $\mathrm{CO}$ is carbon monoxide, $\mathrm{N}_{2} \mathrm{O}$ is nitrous oxide, $\mathrm{NO}_{x}$ means nitrogen oxides, $\mathrm{NMVOC}$ means non-methane volatile organic compound, and $\mathrm{SO}_{2}$ is sulfur dioxide. Source: Eggleston, Simon, Leandro Buendia, Kyoko Miwa, Todd Ngara, and Kyoto Tanabe. 2006. 2006 IPCC Guidelines for National Greenhouse Gas Inventories. IPCC. www.ipcc-nggip.iges.or.jp/ public/2006gl/. 
An illustration of calculations for passenger aviation emissions is provided in Table 8. The table presents two routes: Jakarta (CGK) to Makassar (UPG) and Makassar (UPG) to Kendari (KDI). This is a route to Eastern Indonesia with a transit in Makassar, an Indonesian hub airport.

\section{Table 8 | Sample Calculation for Aviation Passenger Emissions}

\begin{tabular}{|c|c|c|c|c|c|c|c|c|c|c|c|c|c|c|}
\hline \multirow{6}{*}{$\mathrm{CO}_{2}$} & \multirow[b]{2}{*}{$\begin{array}{l}\text { Flight } \\
\text { number }\end{array}$} & \multirow[b]{2}{*}{ Route (D/I)* } & \multirow[b]{2}{*}{ Airline } & \multirow[b]{2}{*}{ Aircraft } & \multicolumn{2}{|c|}{ Emission Factor } & \multirow[b]{2}{*}{$\begin{array}{l}\text { Distance } \\
(\mathrm{km})\end{array}$} & \multirow{2}{*}{$\begin{array}{l}\text { Distance } \\
\text { correction } \\
\text { factor } \\
(\mathrm{km})\end{array}$} & \multirow{2}{*}{$\begin{array}{l}\text { Climb-cruise- } \\
\text { descent fuel } \\
\text { consumption } \\
\text { rate }(\mathrm{kg} / \mathrm{km})\end{array}$} & \multirow[b]{2}{*}{$\begin{array}{l}\text { Trip total emissions } \\
\left(\mathrm{kgCO}_{2}\right)\end{array}$} & \multirow[b]{2}{*}{\begin{tabular}{|l} 
Passenger- \\
to-cargo \\
factor $(\%)$
\end{tabular}} & \multirow[b]{2}{*}{$\begin{array}{l}\text { Seating } \\
\text { capacity } \\
\text { (pax) }\end{array}$} & \multirow[b]{2}{*}{$\begin{array}{l}\text { Passenger } \\
\text { load factor } \\
(\%)\end{array}$} & \multirow[b]{2}{*}{$\begin{array}{l}\text { Emissions per } \\
\text { person (kg CO } / \\
\text { trip) }\end{array}$} \\
\hline & & & & & $\begin{array}{l}\mathrm{CCD}(\mathrm{kg} \\
\mathrm{CO}_{2} / \mathrm{kg} \\
\text { fuel) }\end{array}$ & $\begin{array}{l}\mathrm{LTO}(\mathrm{kg} \\
\left.\mathrm{CO}_{2} / \mathrm{LTO}\right)\end{array}$ & & & & & & & & \\
\hline & (A) & (B) & (C) & (D) & (E) & (F) & (G) & (H) & (I) & $(\mathrm{J})=\left((\mathrm{E})^{*}((\mathrm{G})+(\mathrm{H}))^{*}(\mathrm{I})\right)+(\mathrm{F})$ & $(\mathrm{K})$ & $(\mathrm{L})$ & (M) & $\begin{array}{l}(\mathrm{N})=(\mathrm{J}) *(\mathrm{~K}) / \\
\left((\mathrm{L})^{*}(\mathrm{M})\right)\end{array}$ \\
\hline & 1 & CGK-UPG [D] & Garuda Indonesia & Boeing 737-800NG [m] & 3.15 & 2784 & 1528 & 100 & 3.85 & 22528 & 62.09 & 162 & 74 & 117 \\
\hline & 2 & UPG-KDR [D] & Garuda Indonesia & Boeing 737-800 & 3.15 & 2784 & 376 & 50 & 3.56 & 7561 & 62.09 & 189 & 74 & 34 \\
\hline & \multicolumn{4}{|c|}{ Emissions per person for entire journey ( $\mathrm{kg} \mathrm{CO}_{2} /$ trip) } & & & & & & & & & & 150 \\
\hline \multirow{6}{*}{$\mathrm{CH}_{4}$} & \multirow[b]{2}{*}{$\begin{array}{l}\text { Flight } \\
\text { number }\end{array}$} & \multirow[b]{2}{*}{ Route (D/I)* } & \multirow[b]{2}{*}{ Airline } & \multirow[b]{2}{*}{ Aircraft } & \multicolumn{2}{|c|}{ Emission Factor } & & & & & & & & \\
\hline & & & & & $\begin{array}{l}\mathrm{CCD}(\mathrm{kg} \\
\mathrm{CH}_{4} / \mathrm{kg} \\
\text { fuel) }\end{array}$ & $\begin{array}{l}\mathrm{LTO}(\mathrm{kg} \\
\left.\mathrm{CH}_{4} / \mathrm{LTO}\right)\end{array}$ & \begin{tabular}{|l} 
Distance \\
$(\mathbf{k m})$
\end{tabular} & $\begin{array}{l}\text { correction } \\
\text { factor } \\
(\mathrm{km})\end{array}$ & $\begin{array}{l}\text { descent fuel } \\
\text { consumption } \\
\text { rate }(\mathrm{kg} / \mathrm{km})\end{array}$ & $\begin{array}{l}\text { Trip total emissions } \\
\left(\mathrm{kgCH}_{4}\right)\end{array}$ & $\begin{array}{l}\text { Passenger- } \\
\text { to-cargo } \\
\text { factor (\%) }\end{array}$ & $\begin{array}{l}\text { Seating } \\
\text { capacity } \\
\text { (pax) }\end{array}$ & $\begin{array}{l}\text { Passenger } \\
\text { load factor } \\
\text { (\%) }\end{array}$ & $\begin{array}{l}\text { Emissions per } \\
\text { person }\left(\mathrm{kg} \mathrm{CH}_{4} /\right. \\
\text { trip) }\end{array}$ \\
\hline & (A) & (B) & (C) & (D) & (E) & $(F)$ & (G) & (H) & (I) & $(\mathrm{J})=\left((\mathrm{E})^{*}((\mathrm{G})+(\mathrm{H}))^{*}(\mathrm{I})\right)+(\mathrm{F})$ & $(K)$ & $(\mathrm{L})$ & (M) & $\begin{array}{l}(\mathrm{N})=(\mathrm{J}) *(\mathrm{~K}) / \\
((\mathrm{L}) *(\mathrm{M}))\end{array}$ \\
\hline & 1 & CGK-UPG [D] & Garuda Indonesia & Boeing 737-800NG [m] & 0.02 & 0.07 & 1528 & 100 & 3.85 & 125 & 62.09 & 162 & 74 & 0.6496 \\
\hline & 2 & UPG-KDR [D] & Garuda Indonesia & Boeing 737-800 & 0.02 & 0.07 & 376 & 50 & 3.56 & 30 & 62.09 & 189 & 74 & 0.1350 \\
\hline & Emissions & s per person for & entire journey ( $\mathrm{kg} \mathrm{C}$ & $\mathrm{H}_{4} /$ trip) & & & & & & & & & & 0.7846 \\
\hline & & & & & Emission & Factor & & Distance & Climb-cruise- & & & & & \\
\hline & $\begin{array}{l}\text { Flight } \\
\text { number }\end{array}$ & Route (D/I)* & Airline & Aircraft & $\begin{array}{l}\text { CCD (kg } \\
\text { CO/kg } \\
\text { fuel) }\end{array}$ & $\begin{array}{l}\text { LTO (kg } \\
\text { CO/LTO) }\end{array}$ & $\begin{array}{l}\text { Distance } \\
(\mathbf{k m})\end{array}$ & $\begin{array}{l}\text { correction } \\
\text { factor } \\
(\mathrm{km})\end{array}$ & $\begin{array}{l}\text { descent fuel } \\
\text { consumption } \\
\text { rate }(\mathrm{kg} / \mathrm{km})\end{array}$ & $\begin{array}{l}\text { Trip total emissions } \\
(\mathrm{kgCO})\end{array}$ & $\begin{array}{l}\text { Passenger- } \\
\text { to-cargo } \\
\text { factor (\%) }\end{array}$ & $\begin{array}{l}\text { Seating } \\
\text { capacity } \\
\text { (pax) }\end{array}$ & $\begin{array}{l}\text { Passenger } \\
\text { load factor } \\
\text { (\%) }\end{array}$ & $\begin{array}{l}\text { Emissions per } \\
\text { person (kg CO/ } \\
\text { trip) }\end{array}$ \\
\hline CO & (A) & (B) & (C) & (D) & (E) & (F) & (G) & (H) & (I) & $(\mathrm{J})=\left((\mathrm{E}) *((\mathrm{G})+(\mathrm{H}))^{*}(\mathrm{I})\right)+(\mathrm{F})$ & (K) & (L) & (M) & $\begin{array}{l}(\mathrm{N})=(\mathrm{J}) *(\mathrm{~K}) / \\
\left((\mathrm{L})^{*}(\mathrm{M})\right)\end{array}$ \\
\hline & 1 & CGK-UPG [D] & Garuda Indonesia & Boeing 737-800NG [m] & 7 & 7.07 & 1528 & 100 & 3.85 & 43882 & 62.09 & 162 & 74 & 227.2784 \\
\hline & 2 & UPG-KDR [D] & Garuda Indonesia & Boeing 737-800 & 7 & 7.07 & 376 & 50 & 3.56 & 10623 & 62.09 & 189 & 74 & 47.1601 \\
\hline & Emissions & sper person for & entire journey ( $\mathrm{kg} \mathrm{C}$ & $0 /$ trip) & & & & & & & & & & 274.4385 \\
\hline & & & & & Emission & Factor & & Distance & Climb-cruise- & & Passenger- & & & \\
\hline & $\begin{array}{l}\text { Flight } \\
\text { number }\end{array}$ & Route (D/I)* & Airline & Aircraft & $\begin{array}{l}\mathrm{CCD}(\mathrm{kg} \\
\mathrm{N}_{2} 0 / \mathrm{kg} \\
\text { fuel) }\end{array}$ & $\begin{array}{l}\text { LTO (kg } \\
\left.\mathrm{N}_{2} \mathrm{O} / \mathrm{LTO}\right)\end{array}$ & \begin{tabular}{|l} 
Distance \\
$(\mathbf{k m})$
\end{tabular} & \begin{tabular}{|l|} 
correction \\
factor \\
$(\mathrm{km})$
\end{tabular} & $\begin{array}{l}\text { descent fuel } \\
\text { consumption } \\
\text { rate }(\mathrm{kg} / \mathrm{km})\end{array}$ & $\begin{array}{l}\text { Trip total emissions } \\
\left(\mathrm{kgN}_{2} \mathrm{O}\right)\end{array}$ & $\begin{array}{l}\text { to-cargo } \\
\text { factor } \\
(\%)\end{array}$ & $\begin{array}{l}\text { Seating } \\
\text { capacity } \\
\text { (pax) }\end{array}$ & $\begin{array}{l}\text { Passenger } \\
\text { load factor } \\
\text { (\%) }\end{array}$ & $\begin{array}{l}\text { person ( } \mathrm{kg} \mathrm{N}_{2} \mathrm{O} / \\
\text { trip) }\end{array}$ \\
\hline $\mathrm{N}_{2} \mathrm{O}$ & (A) & (B) & (C) & (D) & (E) & (F) & (G) & (H) & (I) & $(\mathrm{J})=\left((\mathrm{E})^{*}((\mathrm{G})+(\mathrm{H}))^{*}(\mathrm{l})\right)+(\mathrm{F})$ & (K) & (L) & (M) & $\begin{array}{l}(\mathrm{N})=(\mathrm{J}) *(\mathrm{~K}) / \\
\left((\mathrm{L})^{*}(\mathrm{M})\right)\end{array}$ \\
\hline & 1 & CGK-UPG [D] & Garuda Indonesia & Boeing 737-800NG [m] & 0.001 & 0.1 & 1528 & 100 & 3.85 & 6 & 62.09 & 162 & 74 & 0.0330 \\
\hline & 2 & UPG-KDR [D] & Garuda Indonesia & Boeing 737-800 & 0.001 & 0.1 & 376 & 50 & 3.56 & 2 & 62.09 & 189 & 74 & 0.0072 \\
\hline & Emissions & s per person for & entire journey ( $\mathrm{kg} \mathrm{N}$ & $\mathrm{N}_{2} 0 /$ trip) & & & & & & & & & & 0.0402 \\
\hline & & & & & Emission & Factor & & Distance & Climb-cruise- & & Passenger- & & & Emission \\
\hline & $\begin{array}{l}\text { Flight } \\
\text { number }\end{array}$ & Route (D/I)* & Airline & Aircraft & $\begin{array}{l}\text { CCD }(\mathrm{kg} \\
\mathrm{NO}_{\mathrm{\gamma}} / \mathrm{kg} \\
\text { fuel) }\end{array}$ & $\begin{array}{l}\text { LTO }(\mathrm{kg} \\
\text { NO } / \mathrm{LTO})\end{array}$ & $\begin{array}{l}\text { Distance } \\
(\mathrm{km})\end{array}$ & \begin{tabular}{|l|} 
correction \\
factor \\
$(\mathrm{km})$
\end{tabular} & $\begin{array}{l}\text { descent fuel } \\
\text { consumption } \\
\text { rate }(\mathrm{kg} / \mathrm{km})\end{array}$ & $\begin{array}{l}\text { Trip total emissions } \\
\left(\mathrm{kgNO}_{\mathrm{x}}\right)\end{array}$ & \begin{tabular}{|l|} 
to-cargo \\
factor \\
$(\%)$ \\
\end{tabular} & $\begin{array}{l}\text { capacity } \\
\text { (pax) }\end{array}$ & $\begin{array}{l}\text { load factor } \\
(\%)\end{array}$ & $\begin{array}{l}\text { person }\left(\mathrm{kg} \mathrm{NO}_{\mathrm{x}} /\right. \\
\text { trip) }\end{array}$ \\
\hline $\mathrm{NO}_{\mathrm{x}}$ & (A) & (B) & (C) & (D) & (E) & (F) & (G) & (H) & (I) & $(J)=\left((E) *((G)+(H))^{*}(I)\right)+(F)$ & (K) & (L) & (M) & $\begin{array}{l}(\mathrm{N})=(\mathrm{J}) *(\mathrm{~K}) / \\
\left((\mathrm{L})^{*}(\mathrm{M})\right)\end{array}$ \\
\hline & 1 & CGK-UPG [D] & Garuda Indonesia & Boeing 737-800NG [m] & 0.011 & 12.3 & 1528 & 100 & 3.85 & 81 & 62.09 & 162 & 74 & 0.4208 \\
\hline & 2 & UPG-KDR [D] & Garuda Indonesia & Boeing 737-800 & 0.011 & 12.3 & 376 & 50 & 3.56 & 29 & 62.09 & 189 & 74 & 0.1287 \\
\hline & Emissions & s per person for & entire journey ( $\mathrm{kg} \mathrm{N}$ & $\mathrm{N}_{x}$ /trip) & & & & & & & & & & 0.5495 \\
\hline & & & & & Emission & Factor & & Distance & Climb-cruise- & & & & & \\
\hline & $\begin{array}{l}\text { Flight } \\
\text { number }\end{array}$ & Route (D/I)* & Airline & Aircraft & $\begin{array}{l}\text { CCD }(\mathrm{kg} \\
\text { NMVOC/ } \\
\mathrm{kg} \text { fuel) }\end{array}$ & $\begin{array}{l}\text { LTO (kg } \\
\text { NMVOC/ } \\
\text { LTO) }\end{array}$ & $\begin{array}{l}\text { Distance } \\
(\mathrm{km})\end{array}$ & \begin{tabular}{|l} 
correction \\
factor \\
$(\mathrm{km})$
\end{tabular} & $\begin{array}{l}\text { descent fuel } \\
\text { consumption } \\
\text { rate }(\mathrm{kg} / \mathrm{km})\end{array}$ & $\begin{array}{l}\text { Trip total emission } \\
\text { (kgNMVOC) }\end{array}$ & $\begin{array}{l}\text { Passenger- } \\
\text { to-cargo } \\
\text { factor (\%) }\end{array}$ & $\begin{array}{l}\text { Seating } \\
\text { capacity } \\
\text { (Pax) }\end{array}$ & $\begin{array}{l}\text { Passenger } \\
\text { load factor } \\
(\%)\end{array}$ & $\begin{array}{l}\text { EmIssions per } \\
\text { person (kg } \\
\text { NMVOC/trip) }\end{array}$ \\
\hline NMVOC & (A) & (B) & (C) & (D) & (E) & (F) & (G) & $(H)$ & (l) & $(J)=\left((E)^{*}((G)+(H))^{*}(I)\right)+(F)$ & (K) & $(\mathrm{L})$ & (M) & $\begin{array}{l}(\mathrm{N})=(\mathrm{J}) *(\mathrm{~K}) / \\
\left((\mathrm{L})^{*}(\mathrm{M})\right)\end{array}$ \\
\hline & 1 & CGK-UPG [D] & Garuda Indonesia & Boeing 737-800NG [m] & 0.0007 & 0.65 & 1528 & 100 & 3.85 & 5 & 62.09 & 162 & 74 & 0.0261 \\
\hline & 2 & UPG-KDR [D] & Garuda Indonesia & Boeing 737-800 & 0.0007 & 0.65 & 376 & 50 & 3.56 & 2 & 62.09 & 189 & 74 & 0.0076 \\
\hline & Emissions & s per person for & entire journey ( $\mathrm{kg} \mathrm{N}$ & JMVOC/trip) & & & & & & & & & & 0.0337 \\
\hline & & & & & Emission & Factor & & Distance & Climb-cruise- & & & & & \\
\hline & $\begin{array}{l}\text { Flight } \\
\text { number }\end{array}$ & Route (D/I)* & Airline & Aircraft & $\begin{array}{l}\mathrm{CCD}(\mathrm{kg} \\
\mathrm{SO}_{2} / \mathrm{kg} \\
\text { fuel) }\end{array}$ & $\begin{array}{l}\text { LTO (kg } \\
\mathrm{SO}_{2} / \text { LTO) }\end{array}$ & $\begin{array}{l}\text { Distance } \\
(\mathrm{km})\end{array}$ & \begin{tabular}{|l|} 
correction \\
factor \\
$(\mathrm{km})$
\end{tabular} & $\begin{array}{l}\text { descent fuel } \\
\text { consumption } \\
\text { rate }(\mathrm{kg} / \mathrm{km})\end{array}$ & $\begin{array}{l}\text { Trip total emission } \\
\left(\mathrm{kgSO}_{2}\right)\end{array}$ & $\begin{array}{l}\text { Passenger- } \\
\text { to-cargo } \\
\text { factor (\%) }\end{array}$ & $\begin{array}{l}\text { Seating } \\
\text { capacity } \\
\text { (pax) }\end{array}$ & $\begin{array}{l}\text { Passenger } \\
\text { load factor } \\
\text { (\%) }\end{array}$ & $\begin{array}{l}\text { Emissions per } \\
\text { person (kg SO } / \\
\text { trip) }\end{array}$ \\
\hline $\mathrm{SO}_{2}$ & (A) & (B) & (C) & (D) & (E) & $(F)$ & (G) & (H) & (I) & $(J)=\left((E) *((G)+(H))^{*}(I)\right)+(F)$ & (K) & $(\mathrm{L})$ & (M) & $\begin{array}{l}(\mathrm{N})=(\mathrm{J}) *(\mathrm{~K}) / \\
\left((\mathrm{L})^{*}(\mathrm{M})\right)\end{array}$ \\
\hline & 1 & CGK-UPG [D] & Garuda Indonesia & Boeing 737-800NG [m] & 1 & 0.44 & 1528 & 100 & 3.85 & 6268 & 62.09 & 162 & 74 & 32 \\
\hline & 2 & UPG-KDR [D] & Garuda Indonesia & Boeing 737-800 & 1 & 0.44 & 376 & 50 & 3.56 & 1517 & 62.09 & 189 & 74 & 7 \\
\hline & Emissions & s per person for & entire journey ( $\mathrm{kg} \mathrm{S}$ & $\mathrm{O}_{2}$ /trip) & & & & & & & & & & 39 \\
\hline
\end{tabular}

Note: *D=domestic flight, I=international flight; $\mathrm{CCD}$ is climb-cruise-descent; $\mathrm{LTO}$ is landing and take-off period. $\mathrm{CO}_{2}$ is carbon dioxide, $\mathrm{CH}_{4}$ is methane, $\mathrm{CO}$ is carbon monoxide, $\mathrm{N}_{2} \mathrm{O}$ is nitrous oxide, $\mathrm{NO}_{\mathrm{x}}$ means nitrogen oxides, NMVOC means non-methane volatile organic compound, and $\mathrm{SO}_{2}$ is sulfur dioxide.

Source: WRI Indonesia calculations. 


\subsection{Calculating Marine Transport Emissions}

\section{Method: Calculating Greenhouse Gas and Air Pollutant Emissions}

Calculating GHG emissions and air pollutants for marine passenger transport is carried out according to Sims et al. (2015) and the ADEME (Environment and Energy Management Agency 2012). Similarly with aviation, the ADEME also simplifies the data required from users and gathers various determinants for calculating GHG and air pollutant emissions. A fuelbased approach-an emission calculation based on the fuel consumption on every trip-is used. As users input their travel distance, the fuel consumption is calculated by multiplying travel distance with a fuel consumption factor applicable to the vessel type. Then the fuel consumption is multiplied by the emission factors to calculate the total emissions per trip. To convert this into personal emissions, the total emissions per trip are divided by the capacity of the vessel and the average load factor.

The formula for the fuel-based method of calculating emissions of GHGs and air pollutants is as follows:

Total marine emissions per person $\left(T S E P_{p e}\right)$

$=\sum_{i=1}^{N}\left(E F_{\mathrm{e} / a p} x D_{i} x F s_{\mathrm{j}}\left(\frac{P T C_{i j}}{S_{i j} \times A L F_{i}}+\frac{1-P T C_{i j}}{P C_{i} \times V O_{i} \times V F_{i}}\right)\right)$

where $i(\mathrm{i}=1,2, \ldots \mathrm{N})$ is the number of trips $\left(i^{\text {th }}\right) ; j$ is type of vessel; $E F_{e / a p}$ is emission/pollutant (e/ap) factor (tonne/kg (GHG/p)/ton fuel); $D_{i}$ is the total distance in the $i$-trips $(\mathrm{km}) ; F s_{j}$ is fuel consumption factor $(\mathrm{kg}$ fuel $/ \mathrm{km}$ ) of $j$-vessel; $P T C_{i j}$ is the passenger-to-cargo factor or the ratio of total weight of passengers to the payload based on the vessel type ( $j$ ) for the $i^{\text {th }}$ trip; $S_{i j}$ is the total number of seats available based on the vessel type $(j)$ for the $i^{\text {th }}$ trip; and $A L F_{i}$ is average PLF or the ratio of average number of passengers transported; the number of available seats in the vessel for the $i^{\text {th }}$ trip; $P C_{i}$ is the car capacity based on the vessel type ( $j$ ) for the $i^{\text {th }}$ trip; $V O_{i}$ is the vehicle occupancy for people traveling together in a car for the $i^{\text {th }}$ trip; and $V F_{i}$ is the vehicle factor $(\operatorname{car}=1$, motorcycle $=6)$ for the $i^{\text {th }}$ trip. The total emissions/air pollutants per person are the sum of the calculations for every leg of the journey.
Technical Determinant for Calculating GHG and Air Pollutant Emissions

Fuel consumption, load factor, and seating capacity are used to calculate the marine transport GHG emissions and air pollutants. To ensure the results are relevant locally, the authors use the load factor and average vessel capacity from the busiest passenger seaport in Indonesia, Merak-Bakauheni. The port serves more than 18 million passengers per year (Ministry of Transportation 2014) and connects Java with Sumatra. It is located near Greater Jakarta, the nation's economic and industrial center.

Fuel consumption factors are generated based on an equation from the European Environment Agency (2019). This calculates fuel consumption ( $\mathrm{kg}$ fuel $/ \mathrm{km}$ ) based on the total energy consumption of the ship in kilowatts $(\mathrm{kW})$, which is generated by using travel time and power factor (kW hour) divided by trip distance. The data for these calculations are based on data from Indonesia's Ministry of Transportation (2014), which describes route characteristics (i.e., origin and destination, distance, travel time) and type of ship (i.e., weight, etc). Table 9 provides the fuel consumption factor by ship type.

\section{Table 9 | Marine Transport Fuel Consumption Factors}

\begin{tabular}{|l|l|}
\hline $\begin{array}{l}\text { Weight of Ship } \\
\text { (Gross Tonnage) }\end{array}$ & Fuel Consumption Factor (kg fuel/km) \\
\hline$<250$ & 15 \\
\hline $250-500$ & 21 \\
\hline $5000-1000$ & 25 \\
\hline$>1000$ & 53 \\
\hline
\end{tabular}

Sources: WRI Indonesia calculations based on European Environment Agency (2019) and Ministry of Transportation (2014): European Environment Agency. 2019. "EMEP/EEA Air Pollutant Emission Inventory Guidebook 2019 - European Environment Agency." Publication. 2019. www. eea.europa.eu/publications/emep-eea-guidebook-2019; Ministry of Transportation. 2014. "Land Transport in Figure." Ministry of Transportation.

The passenger capacity of the vessel is represented by seating capacity. The average number of passengers in the ship is calculated by multiplying seating capacity with the PLF. The seating capacity numbers come from the Ministry of Transportation (2014), which gives average seating capacity for the busiest passenger seaport routes. Seating capacity and load factor are illustrated in Table 10. The highest average capacity is found for Merak to Bakauheni with 919 persons per vessel, while the lowest is Tanjung Api-Api to Tanjung Kelian with 188 persons per vessel. 
Table 10 | Marine Transport Seating Capacity and Load Factors

\begin{tabular}{|l|l|l|l|l|l|}
\hline Route & $\begin{array}{l}\text { Average Load } \\
\text { Factor (\%) }\end{array}$ & $\begin{array}{l}\text { Average Seating } \\
\text { Capacity }\end{array}$ & $\begin{array}{l}\text { Average Gross } \\
\text { Tonnage }\end{array}$ & $\begin{array}{l}\text { Passenger-to- } \\
\text { Cargo Factor }\end{array}$ & $\begin{array}{l}\text { Vehicle Capacity of } \\
\text { Vessel }\end{array}$ \\
\hline Bajoe-Kolaka & 39.41 & 345 & 511 & 0.51 & 27 \\
\hline Kayangan-Pototano & 28.09 & 288 & 329 & 0.54 & 20 \\
\hline Ketapang-Gilimanuk & 37.91 & 277 & 331 & 0.43 & 30 \\
\hline Lintas Ujung-Kamal & 56.37 & 250 & 116 & 0.80 & 5 \\
\hline Merak-Bakauheni & 33.62 & 919 & 2877 & 0.42 & 102 \\
\hline Padang Bai-Lembar & 34.03 & 241 & 513 & 0.44 & 25 \\
\hline Tanjung Api-Api-Tanjung Kelian & 66.07 & 188 & 186 & 0.45 & 19 \\
\hline Average & 39.36 & 354 & 695 & 0.51 & 33 \\
\hline
\end{tabular}

Source: Ministry of Transportation. 2014. "Land Transport in Figure." Ministry of Transportation.

The average load factor represents the average number of passengers on a vessel divided by its seating capacity, and is calculated afresh for different routes. Data from the Ministry of Transportation (2014) are used to determine the average load factors. The highest average load factor is found for Tanjung Api-Api to Tanjung Kelian (66.07 percent), while the lowest is Kayangan to Pototano (28.09 percent). The average load factor numbers are also summarized in Table 10 . Where route data are unavailable, average values are used.
Determinants of Emissions of Greenhouse Gases and Air Pollutants

As with aviation, emission factors are used to calculate GHG $\left(\mathrm{CO}_{2}\right)$ and air pollutant $\left(\mathrm{CH}_{4}, \mathrm{NO}_{\mathrm{X}}, \mathrm{PM}_{2.5}\right.$, and $\mathrm{SO}_{2}$ ) emissions for marine transport. While the emission factor ratio between GHG and air pollutant emissions and fuel consumption are used for the fuelbased approach, the emission factor ratio between GHG and air pollutant emissions and distance are used for the distance-based approach calculation. Emission factors for $\mathrm{CO}_{2}$ and air pollutants are based on fossil fuel consumption. The emission factors for marine transport are calculated based on ship and fuel type using data from the European Environment Agency (2019) and Kristensen (2012), as shown in Table 11.

Table 11 | Greenhouse Gas and Air Pollutant Emission Factors by Vessel Type

\begin{tabular}{|c|c|c|c|c|c|c|}
\hline \multirow{2}{*}{ Vessel Type } & \multirow{2}{*}{ Fuel Type } & \multicolumn{5}{|c|}{ Emission Factors (kg/kg fuel) } \\
\hline & & $\mathrm{NO}_{\mathrm{x}}^{\mathrm{a}}$ & $\mathrm{CO}^{\mathrm{a}}$ & $\mathrm{SO}_{2}^{\mathrm{a}}$ & $\mathrm{PM}_{2.5}^{\mathrm{a}}$ & $\mathrm{CO}_{2}^{\mathrm{b}}$ \\
\hline \multirow{2}{*}{ Ship } & Marine diesel oil/marine gas oil & 0.0785 & 0.0074 & 0.0200 & 0.0014 & 3.114 \\
\hline & Gasoline & 0.0094 & 0.5739 & 0.0200 & 0.0095 & 2.750 \\
\hline \multirow{3}{*}{ Boat } & Diesel & 0.0384 & 0.0198 & 0.0200 & 0.0046 & 3.206 \\
\hline & Gasoline: 2-stroke & 0.0033 & 0.4810 & 0.0200 & 0.0126 & 3.114 \\
\hline & Gasoline: 4-stroke & 0.0268 & 0.8510 & 0.0200 & 0.1880 & 3.151 \\
\hline
\end{tabular}

Note: $\mathrm{NO}_{\mathrm{x}}$ means nitrogen oxides, $\mathrm{CO}$ is carbon monoxide, $\mathrm{SO}_{2}$ is sulfur dioxide, $\mathrm{PM}_{25}$ is fine particulate matter with a diameter less than 2.5 micrometers, and $\mathrm{CO}_{2}$ is carbon dioxide.

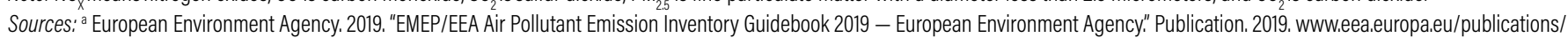
emep-eea-guidebook-2019; ' Kristensen, Hans 0tto. 2012. "Energy Demand and Exhaust Gas Emissions of Marine Engines." Clean Shipping Currents. 
An example of the calculation is shown in Table 12: Merak to Bakauheni route, the busiest passenger route originating in Java.

Table 12 | Sample Calculation for Marine Passenger Emissions

\begin{tabular}{|c|c|c|c|c|c|c|c|c|c|c|c|c|c|}
\hline \multirow{3}{*}{$\mathrm{CO}_{2}$} & Route & $\begin{array}{l}\text { Vehicle (car/ } \\
\text { motorcycle/ } \\
\text { non) }\end{array}$ & \begin{tabular}{|l|} 
Vehicle \\
occupancy \\
(person)
\end{tabular} & $\begin{array}{l}\text { Fuel } \\
\text { type }\end{array}$ & \begin{tabular}{|l|} 
Average gross \\
tonnage (GT)
\end{tabular} & $\begin{array}{l}\text { Emission factor } \\
\left(\mathrm{kg} \mathrm{CO}_{2} / \mathrm{kg} \text { fuel }\right)\end{array}$ & $\begin{array}{l}\text { Distance } \\
(\mathrm{km})\end{array}$ & $\begin{array}{l}\text { Fuel consumption } \\
\text { rate (kg fuel } / \mathrm{km} \text { ) }\end{array}$ & $\begin{array}{l}\text { Passenger- } \\
\text { to-cargo } \\
\text { factor }\end{array}$ & $\begin{array}{l}\text { Seating } \\
\text { capacity } \\
\text { (pax) }\end{array}$ & \begin{tabular}{|l|} 
Average \\
passenger \\
load-factor
\end{tabular} & $\begin{array}{l}\text { Car parking } \\
\text { capacity } \\
\text { (unit) }\end{array}$ & $\begin{array}{l}\text { Vehicle } \\
\text { factor }\end{array}$ \\
\hline & (A) & (B) & (C) & (D) & (E) & (F) & (G) & (H) & (I) & (J) & (K) & (L) & (M) \\
\hline & Merak-Bauheni & Car & 2 & $\begin{array}{l}\text { Marine } \\
\text { diesel oil }\end{array}$ & 2877 & 3.114 & 27.78 & 53 & 0.42 & 919 & 33.62 & 102 & 1 \\
\hline \multirow{3}{*}{ CO } & Route & $\begin{array}{l}\text { Vehicle (car/ } \\
\text { motorcycle/ } \\
\text { non) }\end{array}$ & \begin{tabular}{|l|} 
Vehicle \\
occupancy \\
(person)
\end{tabular} & \begin{tabular}{|l} 
Fuel \\
type
\end{tabular} & \begin{tabular}{|l|} 
Average gross \\
tonnage (GT)
\end{tabular} & $\begin{array}{l}\text { Emission factor } \\
\text { ( } \mathrm{kg} \mathrm{CO} / \mathrm{kg} \text { fuel) }\end{array}$ & \begin{tabular}{|l} 
Distance \\
$(\mathrm{km})$
\end{tabular} & $\begin{array}{l}\text { Fuel consumption } \\
\text { rate (kg fuel } / \mathrm{km} \text { ) }\end{array}$ & $\begin{array}{l}\text { Passenger- } \\
\text { to-cargo } \\
\text { factor }\end{array}$ & $\begin{array}{l}\text { Seating } \\
\text { capacity } \\
\text { (pax) }\end{array}$ & \begin{tabular}{|l|} 
Average \\
passenger \\
load-factor
\end{tabular} & $\begin{array}{l}\text { Car parking } \\
\text { capacity } \\
\text { (unit) }\end{array}$ & $\begin{array}{l}\text { Vehicle } \\
\text { factor }\end{array}$ \\
\hline & (A) & (B) & (C) & (D) & (E) & (F) & (G) & (H) & (I) & (J) & (K) & (L) & (M) \\
\hline & Merak-Bauheni & Car & 2 & $\begin{array}{l}\text { Marine } \\
\text { diesel oil }\end{array}$ & 2877 & 0.0074 & 27.78 & 53 & 0.42 & 919 & 33.62 & 102 & 1 \\
\hline & Route & $\begin{array}{l}\text { Vehicle (car/ } \\
\text { motorcycle/ } \\
\text { non) }\end{array}$ & \begin{tabular}{|l|} 
Vehicle \\
occupancy \\
(person) \\
\end{tabular} & $\begin{array}{l}\text { Fuel } \\
\text { type }\end{array}$ & $\begin{array}{l}\text { Average gross } \\
\text { tonnage (GT) }\end{array}$ & $\begin{array}{l}\text { Emission factor } \\
\text { (kg SO } / 2 \text { / } \mathrm{kg} \text { fuel) }\end{array}$ & $\begin{array}{l}\text { Distance } \\
(\mathrm{km})\end{array}$ & $\begin{array}{l}\text { Fuel consumption } \\
\text { rate (kg fuel/km) }\end{array}$ & $\begin{array}{l}\text { Passenger- } \\
\text { to-cargo } \\
\text { factor }\end{array}$ & $\begin{array}{l}\text { Seating } \\
\text { capacity } \\
\text { (pax) }\end{array}$ & $\begin{array}{l}\text { Average } \\
\text { passenger } \\
\text { load-factor }\end{array}$ & $\begin{array}{l}\text { Car parking } \\
\text { capacity } \\
\text { (unit) }\end{array}$ & $\begin{array}{l}\text { Vehicle } \\
\text { factor }\end{array}$ \\
\hline & (A) & (B) & (C) & (D) & (E) & (F) & (G) & (H) & (I) & (J) & (K) & (L) & (M) \\
\hline & Merak-Bauheni & Car & 2 & $\begin{array}{l}\text { Marine } \\
\text { diesel oil }\end{array}$ & 2877 & 0.02 & 27.78 & 53 & 0.42 & 919 & 33.62 & 102 & 1 \\
\hline \multirow{3}{*}{$\mathrm{PM}_{25}$} & Route & $\begin{array}{l}\text { Vehicle (car/ } \\
\text { motorcycle/ } \\
\text { non) }\end{array}$ & \begin{tabular}{|l|} 
Vehicle \\
occupancy \\
(person)
\end{tabular} & $\begin{array}{l}\text { Fuel } \\
\text { type }\end{array}$ & $\begin{array}{l}\text { Average gross } \\
\text { tonnage (GT) }\end{array}$ & \begin{tabular}{|l|} 
Emission factor \\
$(\mathrm{kg} \mathrm{PM}$ \\
fuel)
\end{tabular} & \begin{tabular}{|l} 
Distance \\
(km)
\end{tabular} & $\begin{array}{l}\text { Fuel consumption } \\
\text { rate (kg fuel/km) }\end{array}$ & $\begin{array}{l}\text { Passenger- } \\
\text { to-cargo } \\
\text { factor }\end{array}$ & $\begin{array}{l}\text { Seating } \\
\text { capacity } \\
\text { (pax) }\end{array}$ & \begin{tabular}{|l|} 
Average \\
passenger \\
load-factor
\end{tabular} & $\begin{array}{l}\text { Car parking } \\
\text { capacity } \\
\text { (unit) }\end{array}$ & $\begin{array}{l}\text { Vehicle } \\
\text { factor }\end{array}$ \\
\hline & (A) & (B) & (C) & (D) & (E) & (F) & (G) & (H) & (I) & (J) & (K) & (L) & (M) \\
\hline & Merak-Bauheni & Car & 2 & $\begin{array}{l}\text { Marrne } \\
\text { diesel oil }\end{array}$ & 2877 & 0.0014 & 27.78 & 53 & 0.42 & 919 & 33.62 & 102 & 1 \\
\hline \multirow{3}{*}{$\mathrm{NO}_{\mathrm{x}}$} & Route & $\begin{array}{l}\text { Vehicle (car/ } \\
\text { motorcycle/ } \\
\text { non) }\end{array}$ & \begin{tabular}{|l|} 
Vehicle \\
occupancy \\
(person)
\end{tabular} & $\begin{array}{l}\text { Fuel } \\
\text { type }\end{array}$ & \begin{tabular}{|l|} 
Average gross \\
tonnage (GT)
\end{tabular} & $\begin{array}{l}\text { Emission factor } \\
\text { (kg NO/ } / \mathrm{kg} \text { fuel) }\end{array}$ & \begin{tabular}{|l} 
Distance \\
$(\mathrm{km})$
\end{tabular} & $\begin{array}{l}\text { Fuel consumption } \\
\text { rate (kg fuel } / \mathrm{km} \text { ) }\end{array}$ & $\begin{array}{l}\text { Passenger- } \\
\text { to-cargo } \\
\text { factor }\end{array}$ & $\begin{array}{l}\text { Seat } \\
\text { capacity } \\
\text { (pax) }\end{array}$ & $\begin{array}{l}\text { Average } \\
\text { passenger } \\
\text { load-factor }\end{array}$ & $\begin{array}{l}\text { Car parking } \\
\text { capacity } \\
\text { (unit) }\end{array}$ & $\begin{array}{l}\text { Vehicle } \\
\text { factor }\end{array}$ \\
\hline & (A) & (B) & (C) & (D) & (E) & (F) & (G) & (H) & (I) & (J) & (K) & (L) & (M) \\
\hline & Merak-Bauheni & Car & 2 & $\begin{array}{l}\text { Marine } \\
\text { diesel oil }\end{array}$ & 2877 & 0.0785 & 27.78 & 53 & 0.42 & 919 & 33.62 & 102 & 1 \\
\hline
\end{tabular}

Note: $\mathrm{CO}_{2}$ is carbon dioxide, $\mathrm{CH}_{4}$ is methane, $\mathrm{CO}$ is carbon monoxide, $\mathrm{N}_{2} \mathrm{O}$ is nitrous oxide, $\mathrm{NO}_{x}$ is nitrogen oxides, $\mathrm{NMVOC}$ is non-methane volatile organic compound, $\mathrm{SO}_{2}$ is sulfur dioxide, and $\mathrm{PM}_{25}$ is fine particulate matter with a diameter less than 2.5 micrometers.

Source: WRI Indonesia calculations

\section{EMISSIONS FOR PERSONAL LOGISTIICS TRANSPORT}

\subsection{Method}

The Guidelines for National Greenhouse Gas

Inventories (Eggleston et al. 2006) classifies emissions from transport activities, including from logistics transport, as emissions from mobile sources. While there are top-down and bottom-up approaches for calculating emissions of GHGs and air pollutants generally, the methodology for calculating personal logistics emissions uses a bottom-up approach. If data are available, the bottom-up approach is preferable, as suggested by the IPCC (Eggleston et al. 2006).

Therefore, EMISI gathers data on the logistics activities of individuals. In this technical note, the authors define personal logistics transport as courier services, both $\mathrm{B} 2 \mathrm{C}$ and $\mathrm{C} 2 \mathrm{C}$, delivering personal goods. This method is specific to e-commerce goods; calculations would be different for transporting heavier goods, such as the bulk transport of coal, cement, or crude oil.
Several parameters are needed to calculate the GHG and air pollutant emissions for personal logistics services. The calculation is illustrated in Figure 2, where the gray boxes represent the process of calculation, the green boxes represent the calculation output, and boxes in other colors represent secondary data. The data needed consist of technical determinants (e.g., mode of transport, capacity of vehicle, distance), data related to the goods to be transported (e.g., type of package, dimensions, and weight), and emission factors. Some data are gathered from users, while others follow parameters from international or Indonesian reports, and others come from interviews with delivery companies (e.g., SiCepat, Ninja Express, Grab). The origin and destination, type of package, dimensions, and weight of the goods are among the data that users input in EMISI.

However, there are complexities in assessing the logistics chain during the journey from origin to destination. The individual has no information about the mode of transport a courier company uses for sending packages, or about the travel distance and mode of transport for each leg. Therefore, this technical note uses a decision tree (see Figure 2) to assess the mode of transport in every leg of a trip. 


\section{USER DATA INPUT FOR PERSONAL LOGISTICS DELIVERY}

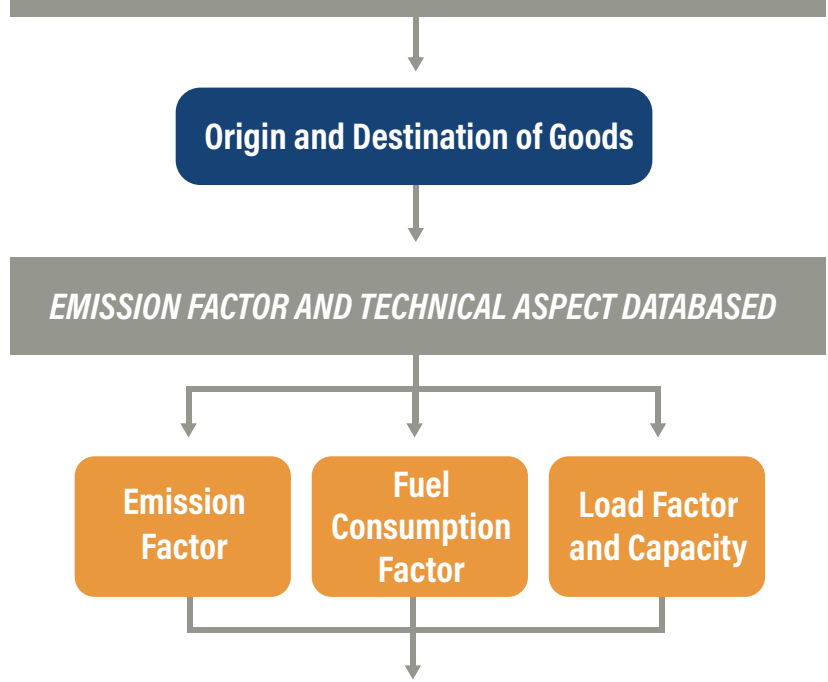

GREENHOUSE GAS EMISSION AND AIR POLLUTANT CALCULATION

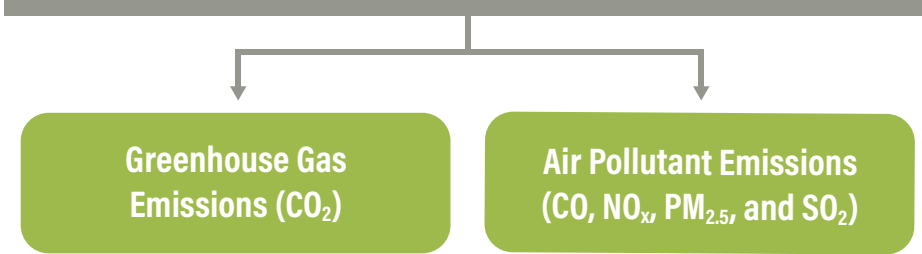

Note: Blue boxes indicate data input, orange are databased and green show output.

Source: WRI Indonesia

\subsection{Determination of Logistics Trip Chain Based on Origin and Destination of Goods}

The decision tree in Figure 3 was developed following interviews with courier companies in Indonesia (e.g., SiCepat, Grab, Ninja Express). The interviews focused on the behavior of operators in managing each delivery (e.g., the combination of several trips to accomplish one journey [the trip chain], choice of mode of transport). Interestingly, most courier companies stated that they use airplanes instead of marine transport for long-distance (intercity and interisland) delivery. The assessment within EMISI is based on the type of delivery service that users input: first, direct services or, second, regular or fast service. With direct services, the courier picks up the package and takes it directly to the recipient. Direct services are usually found for short to medium-length inner-city trips and do not require multiple modes of transport. In Indonesia, direct services are commonly offered by ride-hailing companies such as Grab. Two types of direct service are offered: express/instant, meaning goods ordered by one customer are directly delivered from origin to destination, and regular/fast services, which are same-day services, where multiple packages are carried on one trip. Regular/fast services can be characterized as conventional courier services that require multiple modes of transport for package delivery. This is due to a variety of distance or terrain (e.g., interisland, medium/short intercity distance).

Next, the dimensions and weight of a package are assessed; the package is then categorized as either small or large. The weight and dimensions influence the type of transport used. Motorcycles are used for smaller packages and minibuses for larger. In addition, regular/ fast services are divided by trip distance, while for direct services, distance is not considered. Courier companies (e.g., SiCepat, Ninja Express, Grab, Tiki, Gojek) state that direct/express services commonly serve inner-city movement (Grab 2021) and have a maximum distance they will cover, as they must be completed on the same day. Fast/regular services generally require multiple modes of transport and are divided into truck-based and airplane-based multimodal trips. A marine transportbased multimodal trip is not used in this technical note because most courier companies in Indonesia use airplanes for interisland services. As with fast services, the modes of transport for the first mile and last mile of the trip in fast/regular services are determined based on the dimension/weight of the packages. 


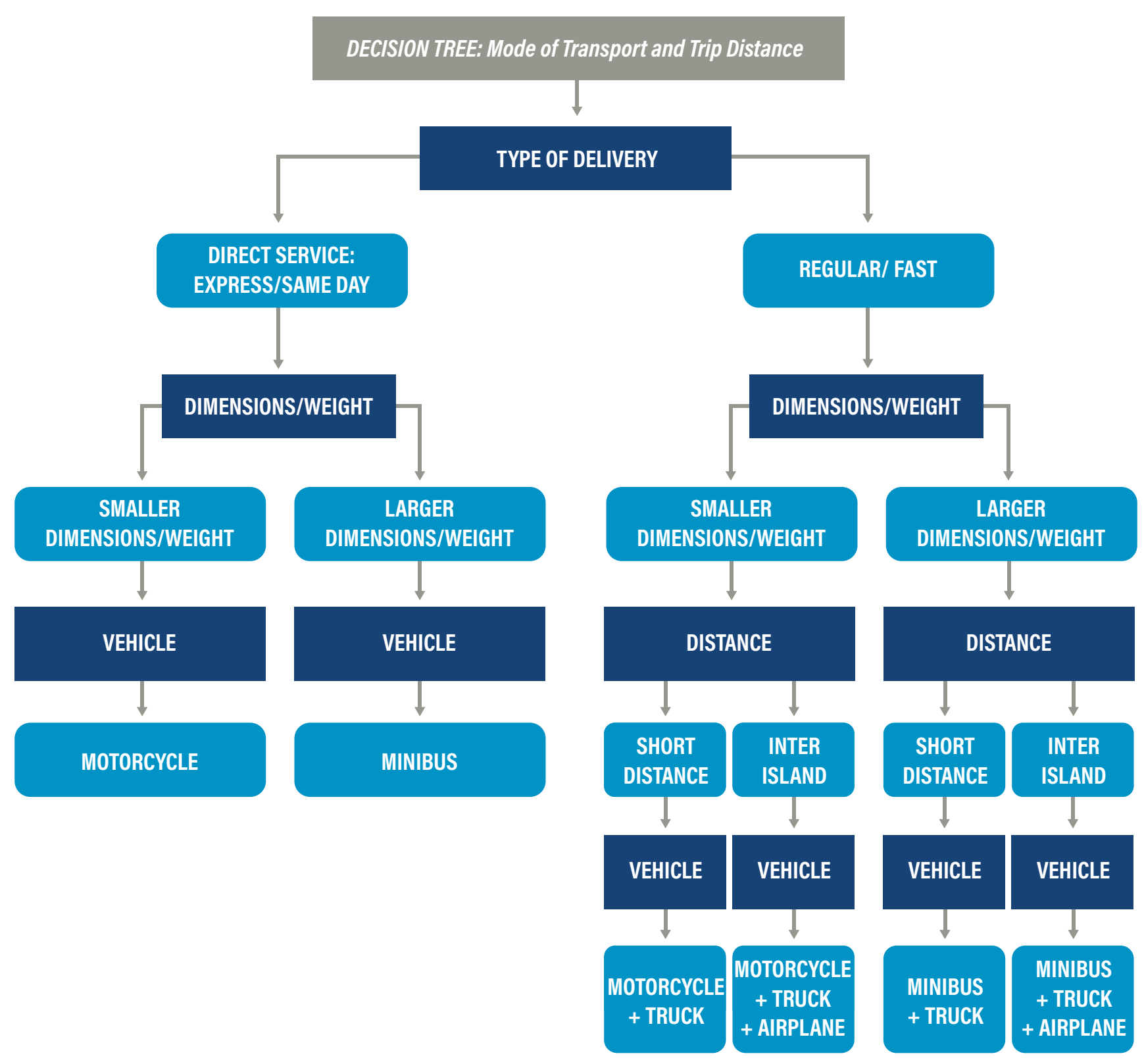

Source: WRI Indonesia, following interviews with delivery companies.

\section{Travel Distance Component}

Two types of trip exist in the delivery of personal goods: first, direct services or express/same day, with a single mode of transport (e.g., motorcycle or minibus), which is dictated by the dimensions and weight of the package; second, regular/fast services, which are commonly served by multiple modes of transport given the trip distance. Bearing in mind those characteristics, the trip distance component was developed. The trip distance for direct services is based on the distance between origin and destination, as assessed by Google Maps. Additional miles must be added for the direct express/same-day and multi-package services, due to the distance a driver must travel to pick up a package (same day) and for extra pickups and drop-offs for other customers (multi-package). Based on interviews with delivery companies, average distance between the driver and the location of each package is $1-2 \mathrm{~km}$. This technical note assumes additional distance $(A)$ of $1.5 \mathrm{~km}$ multiplied by 2 to account for both pickup and drop-off. The additional distance is also applied for express direct services but only for the pickup phase. On the other hand, for regular/fast services, the travel distance from origin and destination must be disaggregated based on the multiple modes of transport used. The illustration of one trip and its components (e.g., distance, modes, and trips) is shown in Figure 4. 


\begin{tabular}{|c|c|c|c|c|c|c|}
\hline \multirow{3}{*}{$\begin{array}{l}\text { Origin } \\
\text { Mode }\end{array}$} & \multicolumn{5}{|c|}{ Direct Services/Express } & \multirow{3}{*}{ Destination } \\
\hline & Additional $A_{1}$ & \multicolumn{4}{|c|}{ Distance $\mathrm{M}_{1}$} & \\
\hline & \multicolumn{5}{|c|}{ Motorcycle/minibus } & \\
\hline \multirow[t]{2}{*}{ Trip } & \multicolumn{5}{|c|}{ Origin - Destination } & \\
\hline & \multicolumn{5}{|c|}{ Direct Services/Same Day } & \\
\hline Origin & Additional $A_{1}$ & & Distance $\mathrm{M}_{1}$ & & Additional $A_{2}$ & Destination \\
\hline Mode & \multicolumn{5}{|c|}{ Motorcycle/minibus } & \\
\hline \multirow[t]{2}{*}{ Trip } & \multicolumn{5}{|c|}{ Origin - Destination } & \multirow{4}{*}{ Destination } \\
\hline & \multicolumn{5}{|c|}{ Regular/Fast Services Inter/Inner City } & \\
\hline Origin & Distance $M_{1}$ & & Distance $\mathrm{M}_{2}$ & & Distance $M_{3}$ & \\
\hline Mode & $\begin{array}{l}\text { Motorcycle/ } \\
\text { minibus }\end{array}$ & & Truck & & $\begin{array}{l}\text { Motorcycle/ } \\
\text { minibus }\end{array}$ & \\
\hline \multirow[t]{2}{*}{ Trip } & $\begin{array}{l}\text { Origin - Courier } \\
\text { Company }\end{array}$ & \multicolumn{3}{|c|}{ Inter Courier Company } & $\begin{array}{l}\text { ourier Company - } \\
\text { Destination }\end{array}$ & \multirow{5}{*}{ Destination } \\
\hline & \multicolumn{5}{|c|}{ Regular/Fast Services Interisland } & \\
\hline Origin & Distance $M_{1}$ & Distance $\mathrm{M}_{2}$ & Distance $\mathbf{M}_{3}$ & Distance $\mathbf{M}_{4}$ & Distance $\mathbf{M}_{5}$ & \\
\hline Mode & $\begin{array}{l}\text { Motorcycle/ } \\
\text { minibus }\end{array}$ & Truck & Airplane & Truck & $\begin{array}{l}\text { Motorcycle/ } \\
\text { minibus }\end{array}$ & \\
\hline Trip & $\begin{array}{l}\text { Origin - Courier } \\
\text { Company }\end{array}$ & $\begin{array}{c}\text { Courier } \\
\text { Company - } \\
\text { Airport }\end{array}$ & $\begin{array}{l}\text { Inter } \\
\text { Airport }\end{array}$ & $\begin{array}{l}\text { Airport - } \\
\text { Courier } \\
\text { Company }\end{array}$ & $\begin{array}{c}\text { Courier } \\
\text { Company - } \\
\text { Destination }\end{array}$ & \\
\hline
\end{tabular}


The total distance of a multimode journey is the sum of the first-mile trip, the trunk trip, and the last-mile trip. The first-mile trip is the distance between the origin point of the parcel to the courier office and the last mile is the distance from the courier office to the destination of the parcel. The trunk trip means the lengthiest leg of the journey, and the modes of transport employed here are usually those with the largest capacity of any on the journey. For an interisland trip, the trunk trip consists of truck and airplane transport, while intercity trips are carried out by truck.

Given that EMISI only collects the distance between origin and destination, the distance of every multimode trip is assumed based on several parameters. Firstly, the first-mile trip from/to home to courier office distance is assumed based on the city. To determine the trip distance the number of courier offices in a city is divided by the area of the city. The outcome represents the area covered by one courier office. The calculation for determining the distance between home and courier office is shown in Table 13. Furthermore, the distance between airports is estimated based on data from Google Maps. The distance between airport and courier company is estimated using total distance between origin and destination, as input by a user, minus the distance between airports and distance between home and courier office.

\section{Table 13 | Average Distance to Courier Company}

\begin{tabular}{ll}
\hline City & Average Distance to Courier Office (km) \\
\hline Bandar Lampung & 0.82 \\
\hline Bandung & 0.48 \\
\hline Batam & 2.37 \\
\hline Jakarta & 0.42 \\
\hline Makassar & 0.85 \\
\hline Surabaya & 0.86 \\
\hline Manado & 2.93 \\
\hline Denpasar & 0.72 \\
\hline Tasikmalaya & 5.41 \\
\hline Medan & 0.77 \\
\hline Padang & 1.49 \\
\hline Palembang & 1.07 \\
\hline Pekanbaru & 1.0 \\
\hline Semarang & 1.09 \\
\hline Balikpapan & 1.66 \\
\hline Ambon & 4.04 \\
\hline Sorong & 13.46 \\
\hline Average & 2.32 \\
\hline
\end{tabular}




\section{Dimension and Weight Classification}

Figure 5 shows a decision tree on how goods are quantified in kilograms by local delivery companies, a system used by local e-commerce outlets. The actual weight and the volumetric weight, as calculated according to the dimension of the goods, are compared. As the capacity of a container is defined not only by the total weight it carries, but also by its volume, a package is quantified based on both weight and dimensions.
However, as weight and dimension use different units, volumetric weight is based on a comparable value between volume and weight of a package. Logistic services in Indonesia define volumetric weight as the product of length $(L ; \mathrm{cm})$, width $(W ; \mathrm{cm})$, and height $(H ; \mathrm{cm})$ of the package, divided by 6,000 , as a converting factor.

Figure 5 | Classifying Weight and Dimensions

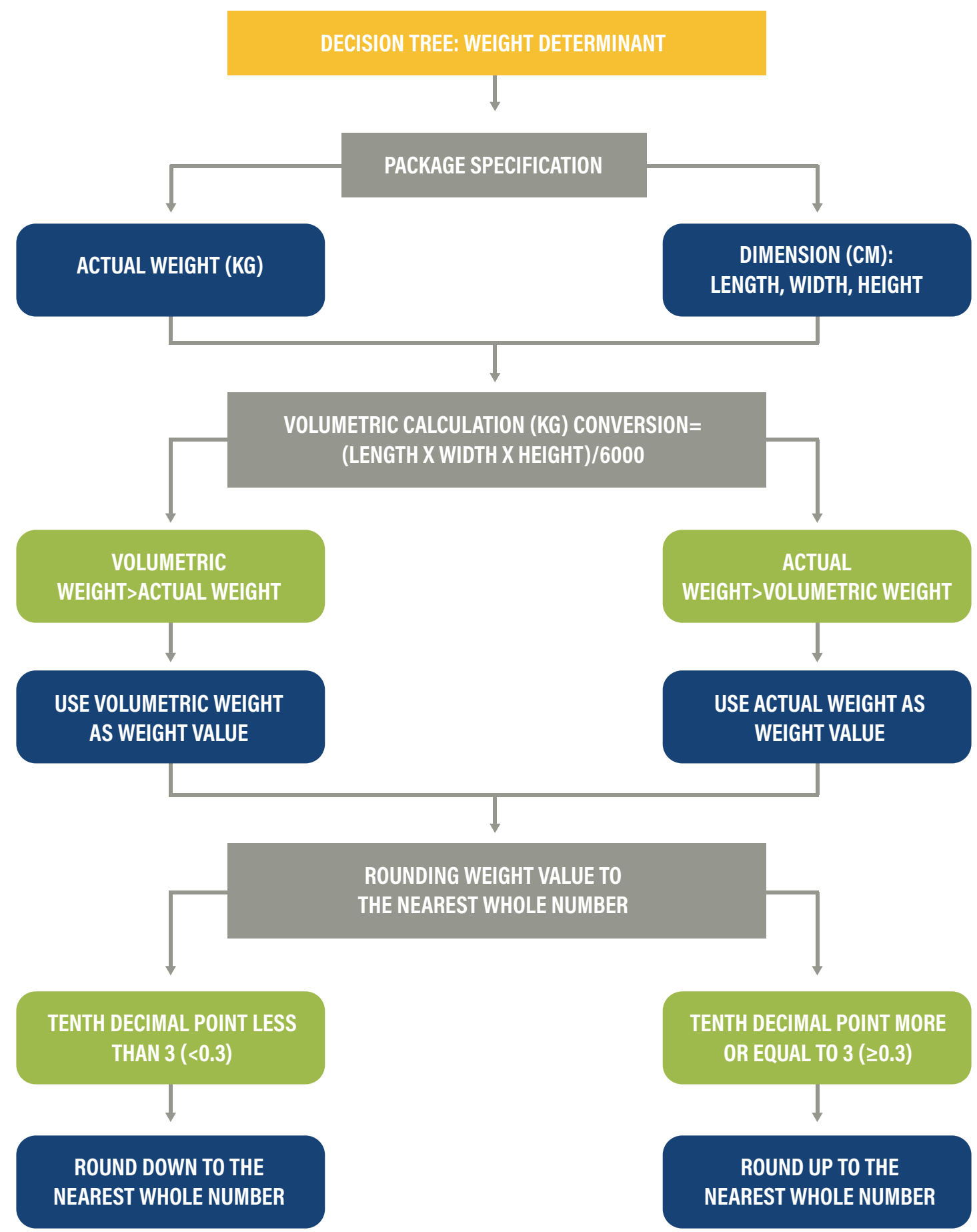

Sources: Tokopedia. 2021. Berat dan Ukuran Paket Benar, Kunci Pengiriman Lancar. https://seller.tokopedia.com/edu/cara-menghitung-berat-paket/; WRI Indonesia. 


\subsection{Calculating Aviation Emissions in Personal Logistics Transport}

\section{Method: Calculating Greenhouse Gas and Air Pollutant Emissions}

Airplanes are commonly used for delivering packages long distances and emissions per person must be calculated. The calculation of the aviation element also uses the fuel-based method, and considers the CCD and LTO, as explained in Section 2.2, "Calculating Emissions from Aviation.”

The formula for calculating GHG and air pollutant emissions from an aviation leg of personal logistics is:

Total aviation logistics emissions per person $\left(T A L E P_{p e}\right)$

$=\sum_{i=1}^{N}\left(\left(\left(E F_{C C D \text { e } / a p} x D c_{i} x F \mathrm{a}_{C C D}\right)+E F_{L T O e / a p}\right) \times \frac{\mathrm{W}}{C_{i j} \times L F_{i}}\right)$

where $i(i=1,2, \ldots N)$ is number of trips $\left(i^{\text {th }}\right)$ captured; $E F_{C C D \text { e/ap }}$ is emission/pollutant (e/ap) factor ( $\mathrm{kg}$ (GHG/p)/kg fuel) during CCD; $D c_{i}$ is the total distance in the $i$-trips $(\mathrm{km})$ corrected with distance correction factor; $F a_{C C D}$ is fuel consumption factor ( $\mathrm{kg}$ fuel $/ \mathrm{km}$ ) during cruise time; $E F_{L T O \text { e/ap }}$ is emission/pollutant (e/ ap) factor ( $\mathrm{kg}(\mathrm{GHG} / \mathrm{p}) / \mathrm{LTO}$ cycle) during LTO; $W$ is the weight of transported goods in kilograms based on weight determination; $\mathrm{C}_{\mathrm{ij}}$ is the capacity of the mode of transport $(j)$ for the $i^{\text {th }}$ trip in kilograms; and LF is the load factor of the mode of transport ( $j$ ) for the $i^{\text {th }}$ trip. The total GHG and air pollutant emissions per person is the sum of the calculations for every leg of the entire journey.

\section{Technical Determinant for Calculating Greenhouse Gas} and Air Pollutant Emissions

The technical determinants for calculating aviation emissions are aircraft characteristics, including fuel consumption during the trip. In contrast to the aviation transport calculation for passengers, the load factor and capacity used are based on the cargo weight. These characteristics are essential for calculating GHG and air pollutant emissions per person. Similar with passenger transport, the fuel consumption for CCD is calculated, this time based on the logistics aircraft Boeing 737-300, which is commonly used by Indonesian airlines for logistics. Table 14 summarizes fuel consumption during CCD based on distance.
Table 14 | Aviation Fuel Consumption during ClimbCruise-Descent

\begin{tabular}{|c|c|}
\hline Distance & Fuel Consumption Rate (kg fuel/km) \\
\hline $125 \mathrm{~nm} \sim 231 \mathrm{~km}$ & 3.95 \\
\hline $250 \mathrm{~nm} \sim 463 \mathrm{~km}$ & 5.74 \\
\hline $500 \mathrm{~nm} \sim 926 \mathrm{~km}$ & 4.03 \\
\hline $750 \mathrm{~nm} 1389 \mathrm{~km}$ & 3.8 \\
\hline $1000 \mathrm{~nm} \sim 1852 \mathrm{~km}$ & 3.64 \\
\hline 1500 nm 2778 km & 3.43 \\
\hline 2000 nm 3704 km & 3.29 \\
\hline Average & 3.98 \\
\hline \multicolumn{2}{|c|}{$\begin{array}{l}\text { Note: } 1 \text { nautical mile }(\mathrm{nm})=1.852 \text { kilometer }(\mathrm{km}) \\
\text { Source: ICA0. 2018. "Airport Air Quality Manual. Doc 9889." wwwicao.int/environmental- } \\
\text { protection/CarbonOffset/Documents/Methodology\%20ICA0\%20Carbon\%20Calculator_v11- } \\
\text { 2018.pdf. }\end{array}$} \\
\hline
\end{tabular}

The cargo load factor expresses the average amount of cargo (by weight and dimensions) carried by an aircraft divided by its capacity. The cargo load factor used is based on the type of aircraft, with information provided by the CEIC (2018). The methodology also uses the cargo capacity, the total cargo in kilograms that can be carried by the aircraft. The capacity is used to calculate the average weight of cargo in the aircraft by multiplying the capacity by the cargo load factor. The cargo capacity numbers use data from the ICAO (2018) for Boeing 737300 (see Table 15).

Table 15 | Capacity and Load Factor of Cargo Airplane

\begin{tabular}{l|l|l}
\hline Aircraft & Capacity (kg) & Load Factor (\%) \\
\hline
\end{tabular}

Boeing 737-300 $17000^{\mathrm{a}}$

Sources: a ICA0. 2018. "Airport Air Quality Manual. Doc 9889." www.icao.int/environmentalprotection/CarbonOffset/Documents/Methodology\%20ICA0\%20Carbon\%20Calculator_v112018.pdf; ' CEIC. 2018. "Indonesia Airline Production: International: Passenger Load Factor." www. ceicdata.com/en/indonesia/airline-production/airline-production-international-passengerload-factor.

\section{Determinants of Emissions of Greenhouse Gases and Air Pollutants}

As the fuel-based method is used for this calculation, emission factors based on fuel consumption are used to calculate emissions of GHGs $\left(\mathrm{CO}_{2}\right.$ and $\left.\mathrm{CH}_{4}\right)$ and air pollutants $\left(\mathrm{NO}_{x}, \mathrm{SO}_{2}\right.$ and NMVOC). These factors express the calculated ratio between GHG and air pollutant emissions and fuel consumption. Emission factors for $\mathrm{CO}_{2}$ are based on fossil fuel consumption. The emission factors used for aviation logistics (see Table 16) are similar to those used for airline passengers in Section 2.2. 


\begin{tabular}{l|l|l}
\hline \multirow{2}{*}{ Greenhouse Gas / Air Pollutant } & Emission Factor \\
\cline { 2 - 3 } & Climb-Cruise-Descent (kg/kg fuel) & Landing-Take-0ff (kg/landing-take-off) \\
\hline $\mathrm{CO}_{2}$ & 3.15 & 2737 \\
$\mathrm{CH}_{4}$ & 0.02 & 0.08 \\
$\mathrm{CO}$ & 7.0 & 6.48 \\
$\mathrm{~N}_{2} \mathrm{O}$ & 0.001 & 0.10 \\
$\mathrm{NO}_{x}$ & 0.011 & 6.98 \\
\hline $\mathrm{NMVOC}$ & 0.0007 & 0.76 \\
\hline $\mathrm{SO}_{2}$ & 1.0 & 0.43 \\
\hline
\end{tabular}

Note: $\mathrm{CO}_{2}$ is carbon dioxide, $\mathrm{CH}_{4}$ is methane, $\mathrm{CO}$ is carbon monoxide, $\mathrm{N}_{2} \mathrm{O}$ is nitrous oxide, $\mathrm{NO}_{x}$ means nitrogen oxides, NMVOC is non-methane volatile organic compound, and $\mathrm{SO}_{2}$ is sulfur dioxide. Source: Eggleston, Simon, Leandro Buendia, Kyoko Miwa, Todd Ngara, and Kyoto Tanabe. 2006. 2006 IPCC Guidelines for National Greenhouse Gas Inventories. IPCC. www.ipcc-nggip.iges.or.jp/ public/2006gl/.

\subsection{Calculating Land Transport Emissions in Personal Logistics}

\section{Method: Calculating Greenhouse Gas and Air Pollutant Emissions}

First, the formula for the fuel-based method to calculate GHG emissions for land transport in the fast services and regular services delivery is:

Fuel - based total land transport delivery emissions per person (FTLDEPp)

$=\sum_{i=1}^{N}\left(E F_{e / a p-i j} \times \frac{F C R_{j i} \times D_{j i} \times W}{C_{i j} \times L F_{i j}}\right)$

where $i(i=1,2, \ldots N)$ is number of trips $\left(i^{\text {th }}\right)$ captured; $j$ is land transport modes (e.g., motorcycle, minibus, or truck); $D_{j i}$ is the distance $(\mathrm{km})$ for the land transport mode $(j)$ for the $i^{\text {th }}$ trip; $E F_{e / a p-i j}$ is emission/pollutant (e/ ap) factor based on the land transport mode $(j)$ in the unit of (kg (GHG)/liter); $F C R_{j i}$ is the fuel consumption rate (liter per $\mathrm{km}$ ) for the transport mode $(j)$ for the $i^{\text {th }}$ trip; $W$ is the weight of transported goods in the unit of kilograms based on weight determination; $C_{i j}$ is the capacity of the transport mode $(j)$ for the $i^{\text {th }}$ trip in the unit of kilograms; and $L F_{i j}$ is the load factor of the transport mode $(j)$ for the $i^{\text {th }}$ trip. The total emissions per package is the sum of emissions for each leg of the trip.

The formula for the distance-based method to calculate air pollutant emissions for land transport for fast and regular deliveries is:

Distance - based total land transport delivery emissions per person (DTLDEPp)

$$
=\sum_{i=1}^{N}\left(E F_{e / a p-i j} \times \frac{D_{i j} \times W}{C_{i j} \times L F_{i j}}\right) \quad \text { (5) }
$$

where $i(i=1,2, \ldots N)$ is number of trips $\left(i^{t h}\right)$ captured; $j$ is land transport modes (e.g., motorcycle, minibus, or truck); $D_{i j}$ is the distance $(\mathrm{km})$ for the land transport mode $(j)$ for the $i^{\text {th }}$ trip; $E F_{e / a p-i j}$ is emission/pollutant ( $e / a p$ ) factor based on the land transport mode $(j)$ for the $i^{\text {th }}$ trip in the unit of ( $\mathrm{kg}$ (pollutant)/distance); $W$ is the weight of transported goods in the unit of kilograms based on weight determination; $\mathrm{C}_{\mathrm{ij}}$ is the capacity of the transport mode $(j)$ for the $i^{\text {th }}$ trip in the unit of kilograms; and LF is the load factor of the transport mode $(j)$ for the $i^{t h}$ trip. The total emissions per goods is the sum of the emissions for each leg of the trip.

For direct services, the fuel-based method is used to calculate GHG emissions, with motorcycle and minibus the main modes of transport used for deliveries. The method for calculating emissions is different than that used for regular/ fast services as direct services are provided exclusively for a limited number of packages; load factor is not used in the calculation, and capacity is based on the number of packages. Direct services are characterized with single modes of transport rather than multiple.

The formula for the fuel-based method to calculate $G H G$ emissions in direct services, meaning express/instant and same-day is:

Fuel - based total express delivery emission per person $\left(F T E E P_{p e}\right)$

$=E F_{e / a p-j} x \frac{F C R_{j} x\left(D_{j}+A_{d}\right)}{C_{j}}$

where $j$ is land transport modes (e.g., motorcycle or minibus); $E F_{e / a p-j}$ is the emission and pollutant factor based on the transport mode $(j)$ in the unit of ( $\mathrm{kg}$ (GHG)/liter fuel); $F C R_{j}$ is the fuel consumption rate (liter per $\mathrm{km}$ ) for the transport mode $(j)$; and $D_{j}$ is the total distance (kilometer) for the transport mode $(j) ; A_{d}$ is the additional distance due to driver have to pick-off and drop-off multiple packages (1.5 km for instant/express and $3 \mathrm{~km}$ for same day); $C_{j}$ is the maximum number of packages transported in a single trip. The maximum capacity for each mode of transport can be seen in Table 17 and Table 18. 
The distance-based method is used to calculate air pollutants in direct services, meaning express/instant and same day:

Distance - based total express delivery emission per person $\left(D T E E P_{p e}\right)$

$=E F_{e / a p-j} x \frac{\left(D_{j}+A_{d}\right)}{C_{j}}$

where $j$ is land transport modes (e.g., motorcycle or minibus); $E F_{e / a p-j}$ is the emission and pollutant factor based on the transport mode $(j)$ in the unit of (kg (pollutant)/distance); and $D_{j}$ is the total distance (kilometer) for the transport mode ( $j$ ); $A_{d}$ is the distance added to account for the driver having to pick up and drop off multiple packages (1.5 km for instant/express and $3 \mathrm{~km}$ for same day); $C_{j}$ is the maximum number of items transported in a single trip. The maximum number of items are assumed, as seen in Table 17 and Table 18, based on the type of service chosen by the user.

\section{Technical Determinant for Calculating Greenhouse Gas} and Air Pollutant Emissions

The technical determinants for calculating land transport emissions are vehicle related and include the fuel consumption for the trip and the capacity of the vehicle. These characteristics are essential for calculating GHG emissions and air pollutants per person. Fuel consumption and capacity of the vehicle are given in four types of vehicle generally used for land transport logistics services, and fuel consumption and capacity are presented for each in Table 17. Fuel consumption means the fuel consumed by the vehicle during the journey which, in this methodology, is based on a regulation issued by the Ministry of Environment and Forestry (2010). The regulation provides the fuel consumption rate based on the vehicle and the factors described in Table 17. However, the load factor for land transport logistics is assumed at 70 percent due to lack of data availability.

Table 17 | Fuel Consumption Factors and Vehicle Capacity for Land Transport

\begin{tabular}{llll} 
& Vehicle & Fuel Consumption Rate (km/l) & Capacity (kg) \\
\hline & Large truck & $44^{\mathrm{a}}$ & $4000^{\mathrm{b}}$ \\
\hline & Small truck & $4^{\mathrm{a}}$ & $2200^{\mathrm{b}}$ \\
\hline
\end{tabular}

Sources: a Ministry of Environment and Forestry. 2010. "Peraturan Menteri Negara Lingkungan Hidup Nomor 12 Tahun 2010 - Hukumonline.Com." Ministry of Environment and Forestry Indonesia. https://m.hukumonline.com/pusatdata/detail/lt4c283ee4c67c5/node/lt511a13750665b/peraturan-menteri-negara-lingkungan-hidup-no-12-tahun-2010-pelaksanaan-pengendalian-pencemaran-udaradi-daerah; ' Deliveree. 2021. "Jasa Ekspedisi Kargo Murah Delivery Jakarta (Dimanapun 2021)." www.deliveree.com/id (blog). 2021. www.deliveree.com/id/jasa-ekspedisi-kargo-murah-delivery-jakarta/; c Astramotor. 2020. "6 Tips Dan Cara Sederhana Merawat Motor Baru, Sangat Mudah Dilakukan." 2020. www.astramotor.co.id/tips-dan-cara-sederhana-merawat-motor-baru-sangat-mudah-dilakukan/.

For motorcycles, the capacity is commonly determined by the type of service. The fact that there is no specific standard or available publication for the goods capacity of motorcycles, combined with the complexity of motorcycle delivery services, means most delivery companies develop safety procedures to ensure drivers do not overload their motorcycles. (Drivers are paid by the number of packages they deliver, and therefore may try to carry too much, while customers tend to underestimate the size of their packages.) Some of these companies provide drivers with equipment, such as large bags, for carrying the goods to increase the efficiency of deliveries. This equipment is very visible for the regular and fast delivery services as well as same-day direct services. The capacity of motorcycle delivery services is provided in Table 18 and is based on interviews with local delivery companies. The express/instant direct services provided more exclusive delivery services for one package, while for same-day direct services the capacity ranges from four to six packages. The regular/ fast services have the highest capacity (30-40 packages) because the companies have a sorting facility. 
Table 18 | Motorcycle Capacity for Deliveries

\begin{tabular}{|l|l|}
\hline Service & $\begin{array}{l}\text { Capacity } \\
\text { (packages) }\end{array}$ \\
\hline Direct (door-to-door) service-express/instant & 1 \\
\hline Direct (door-to-door) service-same day & $4-6$ \\
\hline Regular/fast service & $30-40$ \\
\hline
\end{tabular}

Sources: WRI Indonesia based on interviews with delivery companies.

\section{Determinants of Emissions of Greenhouse Gases and Air Pollutants}

Fuel consumption is calculated by multiplying the fuel consumption factor by distance traveled. The total emissions and air pollutants, consequently, are calculated by multiplying fuel consumption by emission factors. The emission factors are described in Table 19. Like the fuel consumption, emission factors for $\mathrm{CO}_{2}$ are calculated on a fuel-based method; the unit, therefore, is emissions per liter of fuel consumed. Fossil fuel emission factors are calculated by multiplying Indonesian emission factors from the MoEF (2019) and the Ministry of Energy and Mineral Resources (2019) in $\mathrm{kg} \mathrm{CO}_{2} / \mathrm{TJ}$ unit by the heating value ( $\mathrm{TJ} / \mathrm{liter}$ ) of the fuel.

Table 19 | $\mathrm{CO}_{2}$ Emission Factors

\begin{tabular}{|l|l|}
\hline Fuel/Energy & $\mathbf{C O}_{\mathbf{2}}$ Emission Factor $\left(\mathbf{k g ~} \mathbf{C O}_{\mathbf{2}} /\right.$ liter $)$ \\
\hline Automotive diesel oil & 3.26 \\
\hline RON 92 gasoline & 3.08 \\
\hline RON 88 gasoline & 3.11 \\
\hline
\end{tabular}

Sources: Ministry of Energy and Mineral Resources. 2019. "Updated Emission Factor for Energy Sector." Pusat Data dan Informasi, Ministry of Energy and Forestry.

While the $\mathrm{CO}_{2}$ calculation uses the fuel-based method, calculating air pollutants $\left(\mathrm{CO}, \mathrm{NO}_{\mathrm{x}}, \mathrm{PM}_{2.5}\right.$, and $\left.\mathrm{SO}_{2}\right)$ requires the distance-based method. The required data are similar to those needed for the $\mathrm{CO}_{2}$ emission calculation, although the emission factors are based on distance traveled and the mode of transport. The emission factors for air pollutants are obtained from studies conducted by the MoEF (2010), as shown in Table 20. The emission factors from electricity are sourced from Hasan et al. (2012), who distinguish air pollutant emission factors based on the type of fuel used in power plants in Indonesia. The emission factors for $\mathrm{PM}_{2.5}$ and other air pollutants were compiled from Shrestha et al. (2013), who developed the Atmospheric Brown Clouds Emission Inventory Manual for Asian developing countries. Emission factors for vehicles without pollution control devices are adopted for $\mathrm{PM}_{2.5}$. Uncontrolled emission factors tend to be higher than the controlled emission factors since they are acquired from vehicles without air pollution control equipment.
Table 20 | Air Pollutant Emission Factors

\begin{tabular}{|c|c|c|c|c|}
\hline \multirow{2}{*}{$\begin{array}{l}\text { Vehicle } \\
\text { Type }\end{array}$} & \multicolumn{4}{|c|}{ Air Pollutant Factor } \\
\hline & $\mathrm{kg} \mathrm{CO} / \mathrm{km}^{\mathrm{b}}$ & $\mathrm{kg} \mathrm{NO}_{\mathrm{x}} / \mathrm{km}^{\mathrm{b}}$ & $\mathrm{kg} \mathrm{PM}_{25} / \mathrm{km}^{\mathrm{b}}$ & $\mathrm{kg} \mathrm{SO}_{2} / \mathrm{km}^{\mathrm{a}}$ \\
\hline Truck & 0.0045 & 0.0126 & 0.0012 & 0.00093 \\
\hline $\begin{array}{l}\text { Minibus } \\
\text { (gasoline) }\end{array}$ & 0.039 & 0.0011 & 0.00005 & 0.000026 \\
\hline $\begin{array}{l}\text { Minibus } \\
\text { (diesel) }\end{array}$ & 0.0069 & 0.00249 & 0.0005 & 0.00044 \\
\hline Motorcycle & 0.0694 & 0.00029 & 0.00023 & 0.0001 \\
\hline $\begin{array}{l}\text { Note: The heating } \\
\text { carbon monoxide; } \\
\text { diameter less than } \\
\text { Sources: a Ministry } \\
\text { Lingkungan Hidup } \\
\text { Forestry Indonesia } \\
\text { It511al3750665b/p } \\
\text { pengendalian-pen } \\
\text { Rupakheti, M., Perr } \\
\text { Emission Inventory } \\
\text { N. T. K., Permadi, D } \\
\text { greenhouse gases } \\
\text { Research Applicat } \\
\text { edited by Krishna } \\
\text { International Publi }\end{array}$ & $\begin{array}{l}\text { lue for gasoline i } \\
0_{x} \text { means nitroge } \\
2.5 \text { micrometers, a } \\
\text { Environment an } \\
\text { Jomor } 12 \text { Tahun } 2 \\
\text { https://m.hukum } \\
\text { aturan-menteri-n } \\
\text { emaran-udara-di- } \\
\text { adi, D. A., Kanabk } \\
\text { Manual, Nairobi, } K \\
\text { r, Dong, N. P., and } \\
\text { rom crop residue } \\
\text { ns in South and S } \\
\text { asad Vadrevu, To } \\
\text { ing. }\end{array}$ & 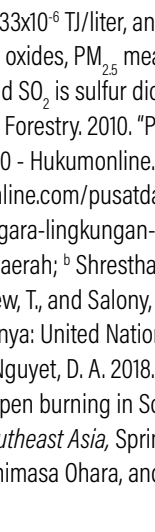 & $\begin{array}{l}\text { or diesel is } 38 \times 10^{-6} \mathrm{~T} \\
\text { fine particulate ma } \\
\text { de. } 1 \mathrm{TJ}=0.2778 \mathrm{GW} \\
\text { turan Menteri Nega } \\
\text { m." Ministry of Envir } \\
\text { /detail//t4c283ee4c } \\
\text { lup-no-12-tahun-201 } \\
\text { M., Kim Oanh, N. T., } \\
\text { 2013. Atmospheric B } \\
\text { nvironmental Progr } \\
\text { mission of toxic air } \\
\text { heast Asia." In Land } \\
\text { er Remote Sensing/ }\end{array}$ & $\begin{array}{l}\text { liter. CO is } \\
\text { er with a } \\
\text { tment and } \\
\text { c5/node/ } \\
\text {-pelaksanaan- } \\
\text { hrestha, } \text { R., }^{\prime} \\
\text { wnn Clouds } \\
\text { nme; }{ }^{\circ} \text { Oanh, } \\
\text { illutants and } \\
\text { tmospheric } \\
\text { hotogrammetry, } \\
\text { am: Springer }\end{array}$ \\
\hline
\end{tabular}

To show the calculations in action, examples are given in Table 21, Table 22, and Table 23 for direct, inter/ inner-city, and interisland journeys, respectively. The sample calculations present three scenarios involving typical personal logistics services (i.e., direct, intercity, and interisland) in Indonesia. The first scenario, described in Table 21, illustrates direct services whereby users send packages expecting delivery within one to two hours, or six to eight hours. Such services are typical of online delivery services attached to the ridehailing apps (e.g., Grab and Gojek) that emerged in 2015. Table 22 describes the second scenario that illustrates the intercity personal logistics services, while the third scenario illustrates the interisland personal logistics services (Table 23). Both scenarios represent the personal courier services that exist currently in Indonesia (i.e., Tiki, SiCepat, Ninja Express, JNT, etc.) 
Table 21 | Sample Calculation for Direct Service Logistics Transport Emissions

\begin{tabular}{|c|c|c|c|c|c|c|c|}
\hline \multirow{3}{*}{$\mathrm{CO}_{2}$} & Type of direct service & $\begin{array}{l}\text { Distance } \\
(\mathrm{km})\end{array}$ & $\begin{array}{l}\text { Additional } \\
\text { distance }(\mathrm{km})\end{array}$ & $\begin{array}{l}\text { Emission } \\
\text { factor } \\
\left(\mathrm{kg} \mathrm{CO}_{2} / \text { liter }\right)\end{array}$ & $\begin{array}{l}\text { Fuel } \\
\text { consumption } \\
\text { rate (km/liter) }\end{array}$ & $\begin{array}{l}\text { Average items } \\
\text { delivered per } \\
\text { trip }\end{array}$ & $\begin{array}{l}\text { Emissions per person } \\
\text { (g CO} 2 \text { /trip) }\end{array}$ \\
\hline & (A) & (B) & (C) & (D) & (E) & $(F)$ & $(\mathrm{G})=\left((\mathrm{D})^{*}((\mathrm{~B})+(\mathrm{C}))\right) /\left((\mathrm{E})^{*}(\mathrm{~F})\right)$ \\
\hline & Motorcycle (1-2 hours delivery) & 20 & 1.5 & 2.68 & 28 & 1 & 2058 \\
\hline \multirow{3}{*}{$\mathrm{CO}$} & Type of direct service & $\begin{array}{l}\text { Distance } \\
(\mathrm{km})\end{array}$ & $\begin{array}{l}\text { Additional } \\
\text { distance (km) }\end{array}$ & \multicolumn{2}{|c|}{$\begin{array}{l}\text { Emission factor } \\
(\mathrm{kg} \mathrm{CO} / \mathrm{km})\end{array}$} & $\begin{array}{l}\text { Average items delivered } \\
\text { per trip }\end{array}$ & $\begin{array}{l}\text { Emissions per person } \\
\text { (g CO/trip) }\end{array}$ \\
\hline & (A) & (B) & (C) & \multicolumn{2}{|l|}{ (D) } & $(E)$ & $(\mathrm{F})=\left((\mathrm{D})^{*}((\mathrm{~B})+(\mathrm{C}))\right) /(\mathrm{E})$ \\
\hline & Motorcycle (1-2 hours delivery) & 20 & 1.5 & \multicolumn{2}{|l|}{0.0694} & 1 & 1492 \\
\hline \multirow[t]{3}{*}{$\mathrm{NO}_{\mathrm{x}}$} & Type of direct service & $\begin{array}{l}\text { Distance } \\
(\mathrm{km})\end{array}$ & $\begin{array}{l}\text { Additional } \\
\text { distance }(\mathrm{km})\end{array}$ & \multicolumn{2}{|c|}{$\begin{array}{l}\text { Emission factor } \\
(\mathrm{kg} \mathrm{NO} / \mathrm{km})\end{array}$} & $\begin{array}{l}\text { Average items delivered } \\
\text { per trip }\end{array}$ & $\begin{array}{l}\text { Emissions per person } \\
\text { (g NO} \mathrm{x}_{\mathrm{x}} \text { /trip) }\end{array}$ \\
\hline & (A) & (B) & (C) & \multicolumn{2}{|l|}{ (D) } & $(E)$ & $(\mathrm{F})=\left((\mathrm{D})^{*}((\mathrm{~B})+(\mathrm{C}))\right) /(\mathrm{E})$ \\
\hline & Motorcycle (1-2 hours delivery) & 20 & 1.5 & \multicolumn{2}{|l|}{0.00029} & 1 & 6.24 \\
\hline \multirow[t]{3}{*}{$\mathrm{PM}_{2.5}$} & Type of direct service & $\begin{array}{l}\text { Distance } \\
(\mathrm{km})\end{array}$ & $\begin{array}{l}\text { Additional } \\
\text { distance }(\mathrm{km})\end{array}$ & \multicolumn{2}{|c|}{$\begin{array}{l}\text { Emission factor } \\
\left(\mathrm{kg} \mathrm{PM}_{2.5} / \mathrm{km}\right)\end{array}$} & $\begin{array}{l}\text { Average items delivered } \\
\text { per trip }\end{array}$ & $\begin{array}{l}\text { Emissions per person } \\
\left(\mathrm{g} \mathrm{PM}_{2.5} / \text { trip) }\right.\end{array}$ \\
\hline & (A) & (B) & (C) & \multicolumn{2}{|l|}{ (D) } & (E) & $(\mathrm{F})=\left((\mathrm{D})^{*}((\mathrm{~B})+(\mathrm{C}))\right) /(\mathrm{E})$ \\
\hline & Motorcycle (1-2 hours delivery) & 20 & 1.5 & \multicolumn{2}{|l|}{0.00023} & 1 & 4.95 \\
\hline \multirow{3}{*}{$\mathrm{SO}_{2}$} & Type of direct service & $\begin{array}{l}\text { Distance } \\
(\mathrm{km})\end{array}$ & $\begin{array}{l}\text { Additional } \\
\text { distance (km) }\end{array}$ & \multicolumn{2}{|c|}{$\begin{array}{l}\text { Emission factor } \\
\left(\mathrm{kg} \mathrm{SO}_{2} / \mathrm{km}\right)\end{array}$} & $\begin{array}{l}\text { Average items delivered } \\
\text { per trip }\end{array}$ & $\begin{array}{l}\text { Emissions per person } \\
\text { (g SO} \mathrm{S}_{2} \text { /trip) }\end{array}$ \\
\hline & (A) & (B) & (C) & \multicolumn{2}{|l|}{ (D) } & $(\mathrm{E})$ & $(\mathrm{F})=\left((\mathrm{D})^{*}((\mathrm{~B})+(\mathrm{C}))\right) /(\mathrm{E})$ \\
\hline & Motorcycle (1-2 hours delivery) & 20 & 1.5 & \multicolumn{2}{|l|}{0.0001} & 1 & 2.15 \\
\hline
\end{tabular}

Note: $\mathrm{CO}_{2}$ is carbon dioxide, $\mathrm{CH}_{4}$ is methane, $\mathrm{CO}$ is carbon monoxide, $\mathrm{N}_{2} \mathrm{O}$ is nitrous oxide, $\mathrm{NO}_{x}$ means nitrogen oxides, $\mathrm{NMVOC}$ means non-methane volatile organic compound, $\mathrm{SO}$ is sulfur dioxide, and $\mathrm{PM}_{25}$ is fine particulate matter with a diameter less than 2.5 micrometers.

Source: WRI Indonesia calculations. 
Table 22 | Calculation Sample for Inter/Inner-City Service Logistics Transport Emissions

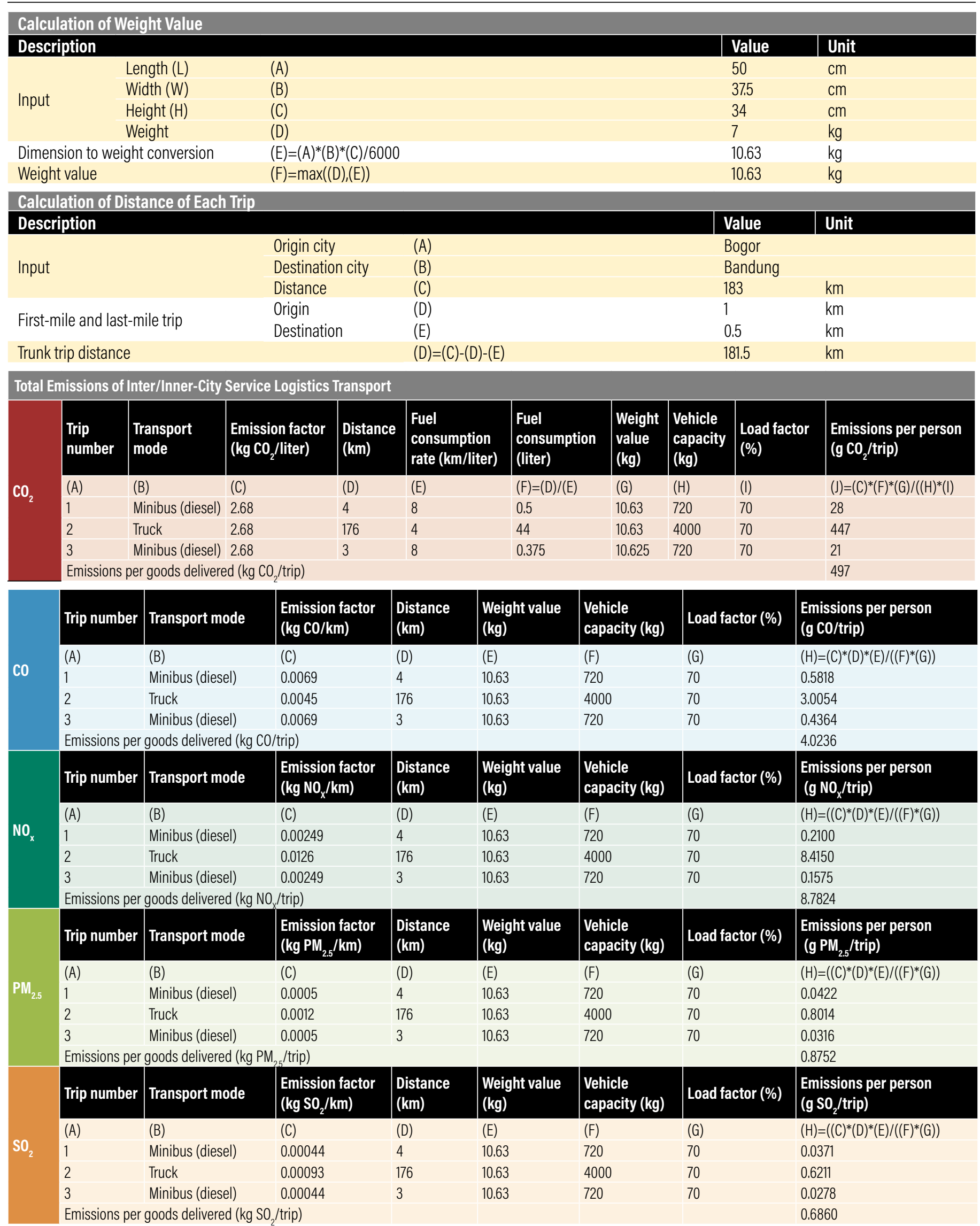

Note: $\mathrm{CO}_{2}$ is carbon dioxide, $\mathrm{CH}_{4}$ is methane, $\mathrm{CO}$ is carbon monoxide, $\mathrm{N}_{2} \mathrm{O}$ is nitrous oxide, $\mathrm{NO}_{\mathrm{X}}$ means nitrogen oxides, $\mathrm{NMVOC}$ means non-methane volatile organic compound, $\mathrm{SO}_{2}$ is sulfur dioxide, and $\mathrm{PM}_{25}$ is fine particulate matter with a diameter less than 2.5 micrometers.

Source: WRI Indonesia calculations 
Table 23 | Sample Calculation for Interisland Service Logistics Transport Emissions

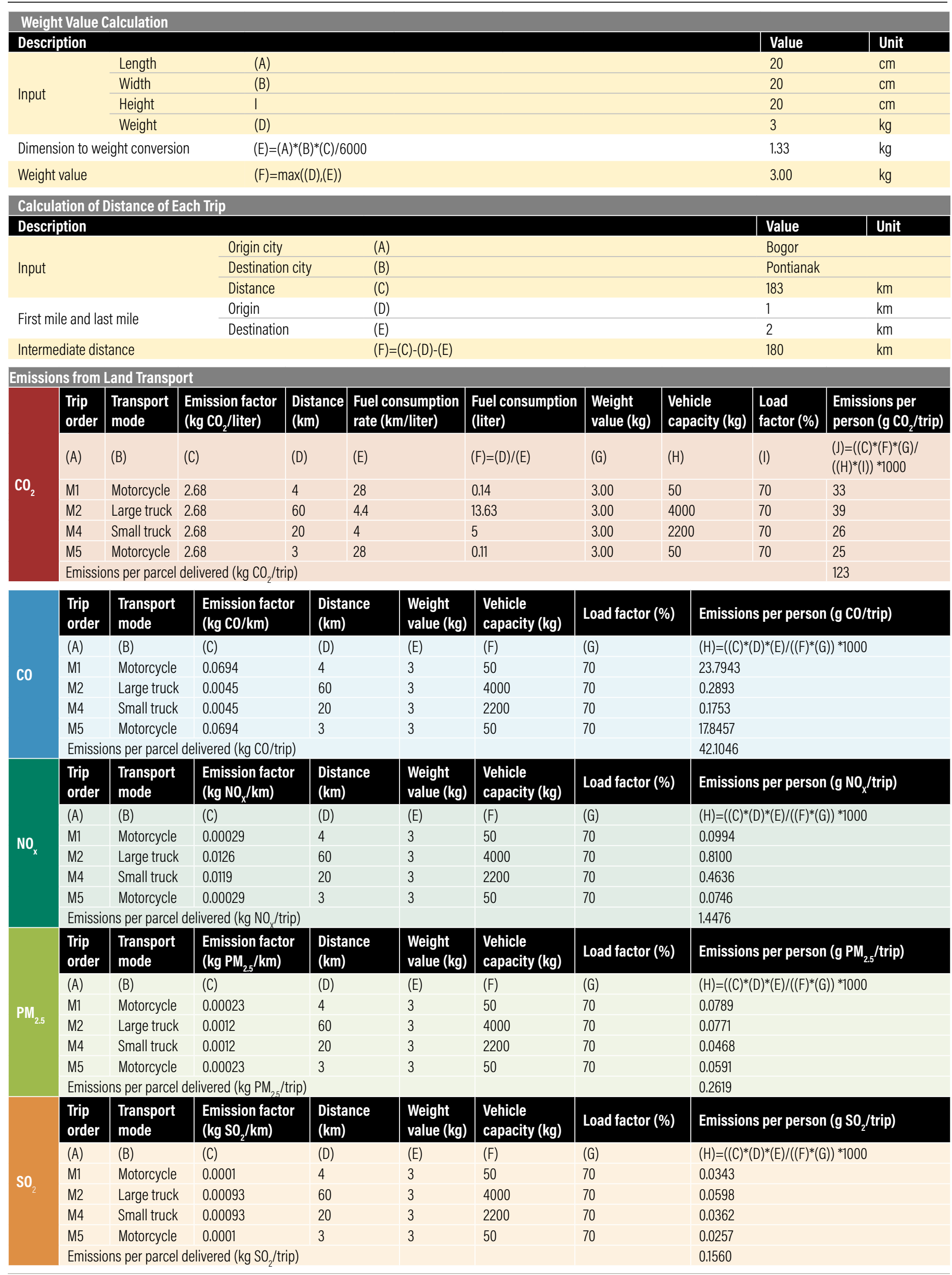




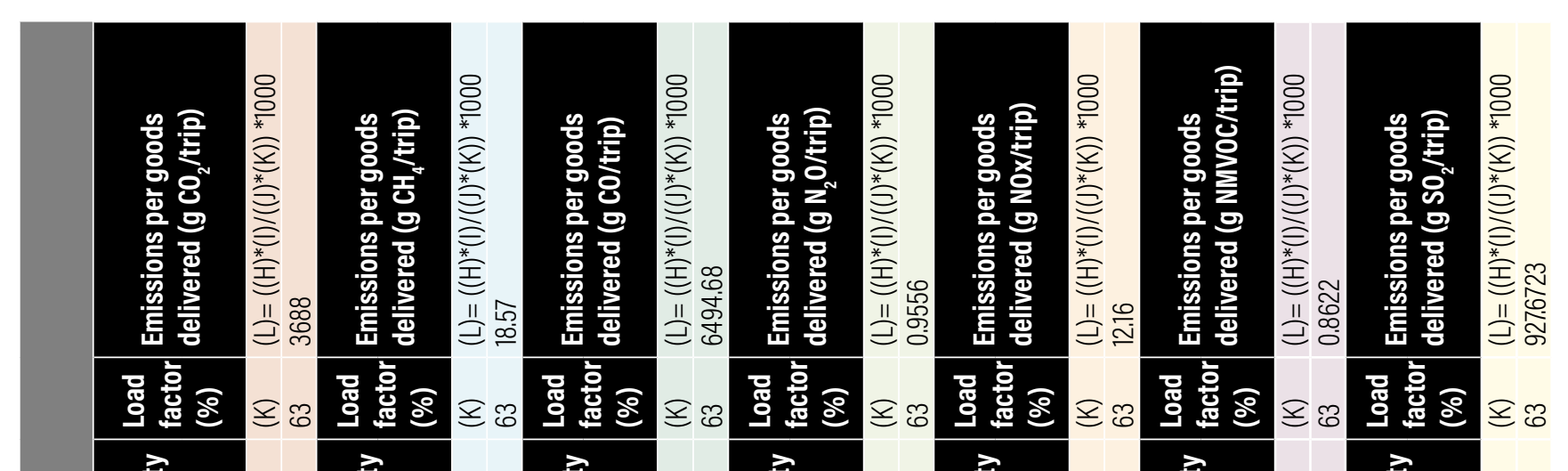

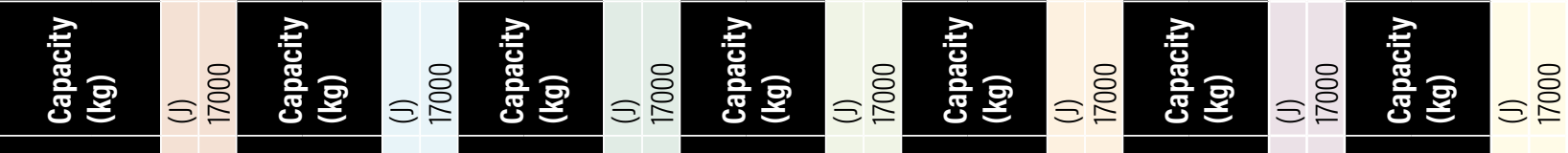

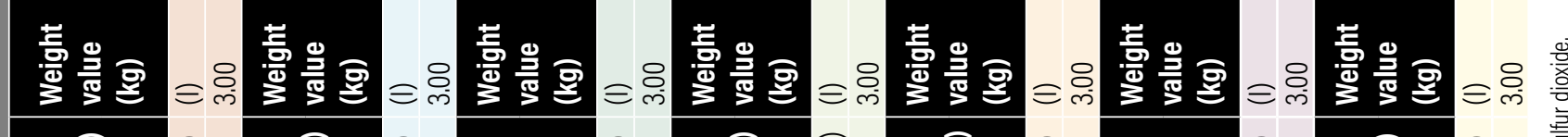

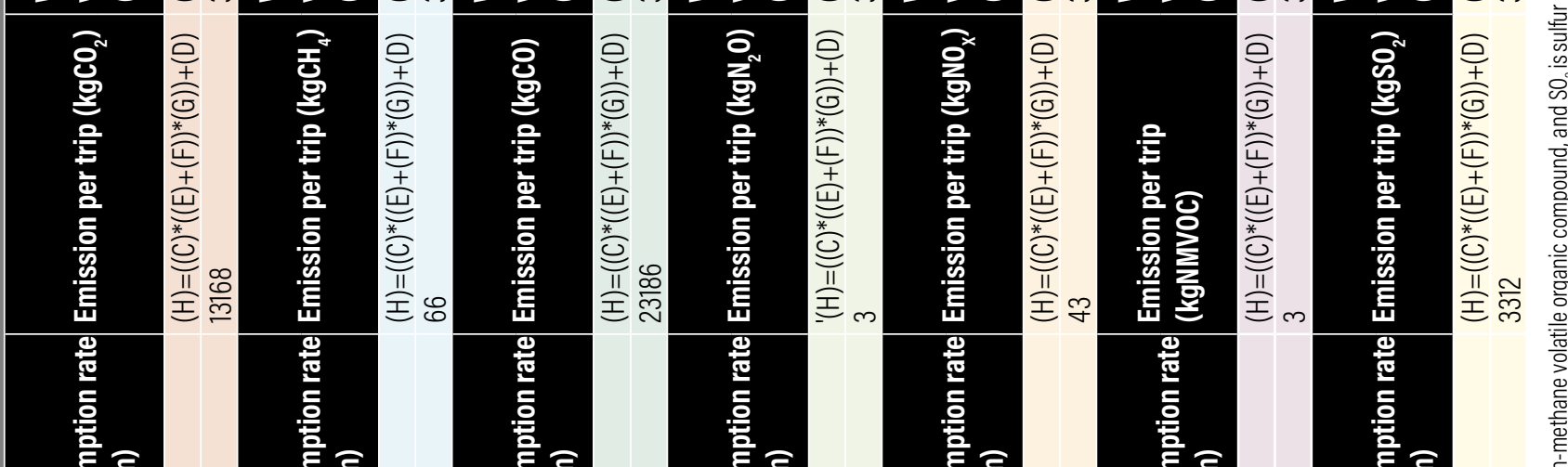

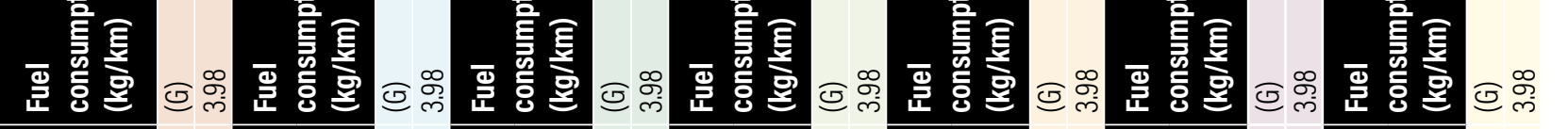

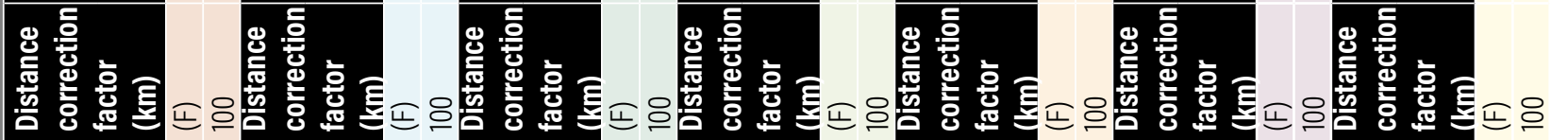

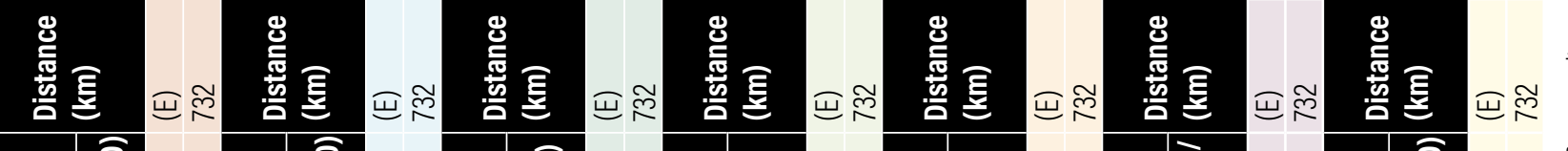
尊

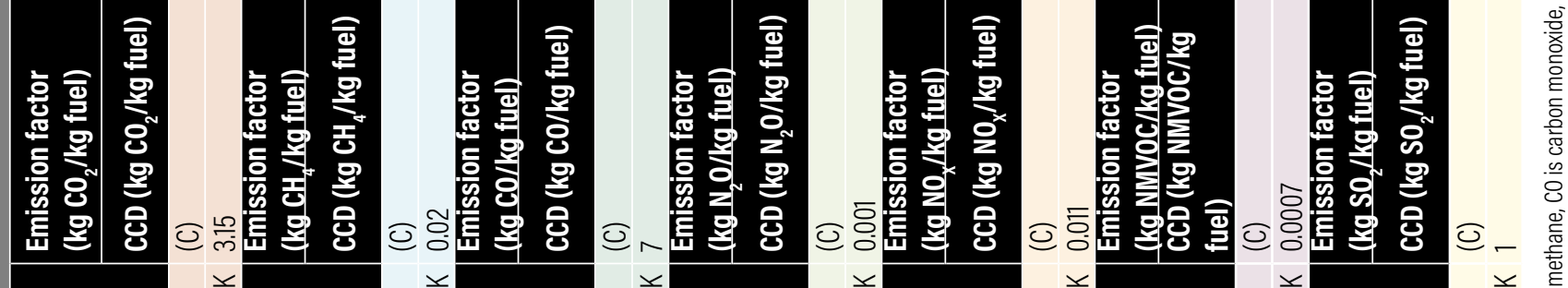

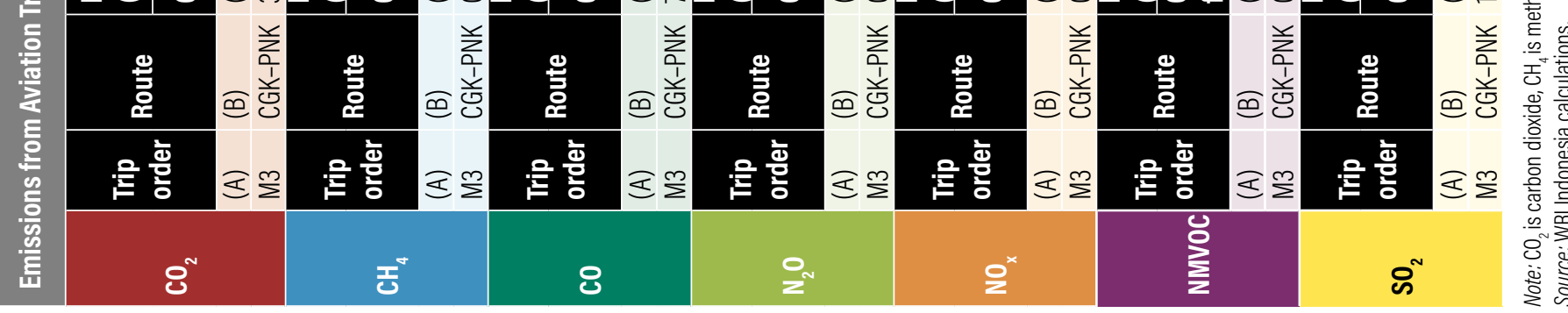




\begin{tabular}{|c|c|c|c|c|c|c|c|c|c|}
\hline \multicolumn{10}{|c|}{ Summary } \\
\hline \multirow{2}{*}{$\begin{array}{l}\text { Trip } \\
\text { order }\end{array}$} & \multirow{2}{*}{ Mode of transport } & \multicolumn{8}{|c|}{ Emissions (gram) } \\
\hline & & $\mathrm{CO}_{2}$ & $\mathrm{CH}_{4}$ & $\mathrm{CO}$ & $\mathrm{N}_{2} \mathrm{O}$ & $\mathrm{NO}_{\mathrm{x}}$ & NMVOC & $\mathrm{PM}_{2.5}$ & $\mathrm{SO}_{2}$ \\
\hline (A) & (B) & (C) & (D) & $(E)$ & $(F)$ & (G) & $(H)$ & (I) & $(J)$ \\
\hline 1 & Motorcycle & 33 & & 23.7943 & & 0.0994 & & 0.0789 & 0.0343 \\
\hline 2 & Large truck & 39 & & 0.2893 & & 0.8100 & & 0.0771 & 0.0598 \\
\hline 3 & Airplane & 3688 & 18.57 & 6494.68 & 0.96 & 12.16 & 0.86 & & 927.67 \\
\hline 4 & Small truck & 26 & & 0.1753 & & 0.4636 & & 0.0468 & 0.0362 \\
\hline 5 & Motorcycle & 25 & & 17.8457 & & 0.0746 & & 0.0591 & 0.0257 \\
\hline \multicolumn{2}{|c|}{ Total emission (grams) } & 3811 & 18.5734 & 6536.7825 & 0.9556 & 13.6059 & 0.8622 & 0.2619 & 927.83 \\
\hline
\end{tabular}

Note: $\mathrm{CO}_{2}$ is carbon dioxide, $\mathrm{CH}_{4}$ is methane, $\mathrm{CO}$ is carbon monoxide, $\mathrm{N}_{2} \mathrm{O}$ is nitrous oxide, $\mathrm{NO}_{x}$ means nitrogen oxides, NMVOC means non-methane volatile organic compound, $\mathrm{SO}$ is sulfur dioxide, and $\mathrm{PM}_{2.5}$ is fine particulate matter with a diameter less than 2.5 micrometers.

\section{LIMITATIONS}

The EMISI app uses the best methods and best available data for calculating emissions in the Indonesian context. While there are several methods provided by the IPCC, ranging from simple to advanced calculations, EMISI tries to balance simple data inputs from users and the complexities of methodology. Most of the calculations for personal aviation, marine, and logistics emissions use Indonesia-specific factors, especially for technical determinants (i.e., capacity, load factors, fuel consumption). The methodology focuses on developing the best estimates and, when local data are unavailable, uses data from global contexts to develop next-best estimates. The calculations in this technical note achieve numbers that are relatively consistent with results from other calculators, especially for countries with similar characteristics to Indonesia. For example, when comparing the $\mathrm{CO}_{2}$ emissions of an airline passenger from Jakarta (CGK) to Makassar (UPG), the difference between the results using the calculations in this technical note and ICAO calculators is insignificant (2.7 percent). The slight difference is due to the fact that emission factors, capacity, and load factors used in this technical note are based on data for Indonesia. However, given the conditions in Indonesia, the methodology is also subject to some limitations as explained in this section. Some prominent literature on calculating aviation air pollution (e.g., Eggleston et al. 2006 and European Environment Agency 2019) presents methods for calculating other significant air pollutants (e.g., $\mathrm{PM}_{2.5}$ ). These, however, require air pollution emission factors from local data, which are unavailable for Indonesia.
Developing locally relevant tools depends substantially on the availability of data and the existing conditions in the country (i.e., technology, materials, behavior). Unfortunately, although the methodology itself is not overly complicated, data availability is the key barrier for the calculation in developing countries, as identified by Song (2017). While some of the factors used in this note have been provided by studies conducted by Indonesian stakeholders (i.e., Ministry of Transport, Ministry of Environment and Forestry, Ministry of Energy and Mineral Resources, airlines, and marine services companies), up-to-date factors are still limited. Recent and upcoming research data on the determinants for calculating emissions in Indonesia-specific cases, such as emission factors and fuel consumption, will improve future calculations. These studies also encourage more detailed and localized data to be produced for the determinants due to demographic, economic, and infrastructure disparities across nations, which affect behavior and thus influence capacity, load factor, fuel consumption factor, and consequently the results of the emissions calculation. Moreover, for direct services of personal logistics, weight and dimensions are not taken into account because the car or motorcycle will carry only one parcel, even though it could carry more. 
Specifically for personal logistics calculations, the assumption on the chain of modes of transport used during deliveries relies on characteristics of the courier industry. In this technical note, as the distance input by users is the distance from origin to destination of the goods, disaggregation of distance is achieved using the framework for courier logistics behavior (Figure 4). The assumption of the first and last mile is the distance from the origin to the nearest package drop-off point. This distance is determined based on the radius of the catchment area, which is calculated based on the number of drop-off points for packages in cities in Indonesia. In addition, the chain of modes of transport used during the journey is dependent on company policy, which is sensitive to government policy (i.e., price, tax, etc.) or availability of those modes. Therefore, it is important to have up-to-date numbers for the first and last miles and the decision tree to increase the reliability of the calculation. Moreover, the personal logistic calculation is limited to domestic deliveries, as data on the international delivery logistic chains are unavailable.

The methodology documented in this technical note will form the basis of the next EMISI update. The EMISI app aims to educate individuals about their carbon footprint and empower them to take actions to protect the climate. The methodology can be adopted by various stakeholders in Indonesia, including government, nongovernmental and private organizations, scientific experts, communities, and individuals. Opportunities to integrate the calculator with current transport apps, public campaigns, and crowdfunding platforms are also being explored. Data resulting from calculations derived from this technical note could also help improve Indonesia's emissions inventory knowledge at the individual level, as well as add value to other research and development activities. 


\section{ABBREVIATIONS}

$\mathrm{B} 2 \mathrm{C}$

business to customer

$\mathrm{C} 2 \mathrm{C}$

customer to customer

CCD climb-cruise-descent

CO carbon monoxide

$\mathrm{CO}_{2} \quad$ carbon dioxide

$\mathrm{CH}_{4} \quad$ methane

EF emission factor

EMISI Indonesia Zero Emissions Application

f function

FCR fuel consumption rate

GHG greenhouse gas

ICAO International Civil Aviation Organization

ICT Information and Communication Technology

IPCC Intergovernmental Panel on Climate Change

LF load factor

LTO landing and take-off period

NMVOC non-methane volatile organic compound

PLF passenger load factor

$\mathrm{PM}_{2.5} \quad$ particulate matter with diameter less than 2.5 micrometers

$\mathrm{SO}_{2} \quad$ sulfur dioxide

\section{ENDNOTES}

1 For the explanation of ro-ro services see: https://www.

marineinsight.com/types-of-ships/what-are-ro-ro-ships/. 


\section{REFERENCES}

Abdelrhim, Mansour, and Abdullah Elsayed. 2020. "The Effect of COVID-19 Spread on the E-Commerce Market: The Case of the 5 Largest E-Commerce Companies in the World," SSRN Scholarly Paper ID 3621166. Rochester, NY: Social Science Research Network. https://doi.org/10.2139/ssrn.3621166.

Amalia, Mia, Budy P. Resosudarmo, and Jeff Bennett. 2013. "The Consequences of Urban Air Pollution for Child Health: What Does Self Reporting Data in the Jakarta Metropolitan Area Reveal?" 2013-09. Departmental Working Papers. The Australian National University, Arndt-Corden Department of Economics. https://ideas,repec.org/p/pas/papers/2013-09.html.

Astramotor. 2020. "6 Tips Dan Cara Sederhana Merawat Motor Baru, Sangat Mudah Dilakukan." www.astramotor.co.id/tips-dan-cara-sederhana-merawatmotor-baru-sangat-mudah-dilakukan/.

Bhwana, Petir Garda. 2020. "Govt's Rp293-Trillion Social Safety Net Cluster Funds to Reduce Burden." Tempo. September 27. https://en.tempo.co/ read/1390555/govts-rp293-trillion-social-safety-net-cluster-funds-to-reduceburden.

Casanova, Albert M., Bert van Leeuwen, Coen Capelle, Simon Finn, and Steven Guo. 2017, "An Overview of Commercial Aircraft 2018-2019." Schipol/London: DVB Bank. www.dvbbank.com/ /media/Files/D/dvbbank-corp/aviation/dvboverview-of-commercial-aircraft-2018-2019.pdf.

CEIC. 2018, "Indonesia Airline Production: International: Passenger Load Factor." www.ceicdata.com/en/indonesia/airline-production/airline-productioninternational-passenger-load-factor.

CEIC. 2020. Indonesia Airline Production. CEIC. www.ceicdata.com/en/ indonesia/airline-production.

Clement, J. 2020. "COVID-19 Impact Retail e-Commerce Site Traffic 2020."

Statista. June 3. www.statista.com/statistics/1112595/covid-19-impact-retail-ecommerce-site-traffic-global/.

Das, Kaushik, Michael Gryseels, Priyanka Sudhir, and Khoon T Tan. 2016. "Unlocking Indonesia's Digital Opportunity." McKinsey \& Company. www. mckinsey.com/featured-insights/asia-pacific/unlocking-indonesias-digitalopportunity.

Deliveree. 2021. "Jasa Ekspedisi Kargo Murah Delivery Jakarta (Dimanapun 2021)." www.deliveree.com/id (blog). www.deliveree.com/id/jasa-ekspedisikargo-murah-delivery-jakarta/.

Eggleston, Simon, Leandro Buendia, Kyoko Miwa, Todd Ngara, and Kyoto Tanabe. 2006. 2006 IPCC Guidelines for National Greenhouse Gas Inventories. IPCC. www.ipcc-nggip.iges.or.jp/public/2006gl/.
Environment and Energy Management Agency, 2012. "CO Information for Transport Services: Methodological Guide." Application of Article L. 1431-3 of the French transport code.

European Environment Agency. 2019. "EMEP/EEA Air Pollutant Emission Inventory Guidebook 2019 - European Environment Agency." Publication. www.eea.europa.eu/publications/emep-eea-guidebook-2019.

Garuda Indonesia. 2020. "Revitalisasi Armada." 2020. www.garudaindonesia.com/id/id/garuda-indonesia-experience/fleets/fleetrevitalization

Grab. 2021. "GrabExpress Same Day Parcel \& Courier Delivery Service." Grab ID. www.grab.com/id/en/express/.

Hasan, M. H., W. K. Muzammil, T. M. I. Mahlia, A. Jannifar, and Hasanuddin, I. 2012. "A Review on the Pattern of Electricity Generation and Emission in Indonesia from 1987 to 2009." Renewable and Sustainable Energy Reviews 16(5): 3206-19.

Hilman, Oliver, and Fiona Harvey. 2019. "US Is Hotbed of Climate Change Denial, Major Global Survey Finds." The Guardian, May 8, sec. Environment. www.theguardian.com/environment/2019/may/07/us-hotbed-climatechange-denial-international-poll.

ICA0 (International Civil Aviation Organization). 2016. "ICA0 Carbon Emissions Calculator." 2016. www.icao.int/environmental-protection/ Carbonoffset/Pages/default.aspx.

ICA0. 2018. "Airport Air Quality Manual. Doc 9889" www.icao.int/ environmental-protection/CarbonOffset/Documents/Methodology\%20 ICA0\%20Carbon\%20Calculator_v11-2018.pdf.

ICA0. 2020. "ICA0 Carbon Emissions Calculator Methodology." www.icao.int/ publications/Documents/9889 cons en.pdf.

Indonesian Bureau of Statistics. 2010. Transportation Statistics 2010. Indonesian Bureau of Statisics. www.bps.go.id/publication/2011/12/01/ fa2236ff14cdbbced1174712/statistik-transportasi-2010.html.

Indonesian Bureau of Statistics. 2019. Sea Transport Statistics 2019. Indonesia: Indonesian Bureau of Statistics. www.bps.go.id/ publication/2020/11/20/a13c82a0a9f343720404cf45/statistik-transportasilaut-2019.html.

IPCC. 2018. "Summary for Policymakers of IPCC Special Report on Global Warming of $1.5^{\circ} \mathrm{C}$ Approved by Governments - IPCC." wwwipcC. ch/2018/10/08/summary-for-policymakers-of-ipcc-special-report-onglobal-warming-of-1-5c-approved-by-governments/. 
Jakarta Post. 2021. "Indonesian Logistics Firms Ride E-Commerce Boom to Survive Pandemic." April 18. www.thejakartapost.com/news/2021/04/18/ indonesian-logistics-firms-ride-e-commerce-boom-to-survive-pandemic.html.

Kristensen, Hans 0tto. 2012، "Energy Demand and Exhaust Gas Emissions of Marine Engines." Clean Shipping Currents 1: 18--26.

Leung, Kang Hang. 2016. Indonesia's Summary Transport Assessment. Asian Development Bank. www.adb.org/publications/indonesia-summary-transportassessment.

Lindau, Toni. 2015. "Transport Plays a Key Role in Urban Air Quality," February. www.wri.org/insights/transport-plays-key-role-urban-air-quality.

Lion Air. 2020. "Armada Kami." www.lionair.co.id/tentang-kami/armada-kami. Husaini, Azis. 2020. "Batik Air, maskapai pesaing Garuda yang kini telah operasikan 76 pesawat," kontan.co.id. May 9. http://industri.kontan.co.id/news/ batik-air-maskapai-pesaing-garuda-yang-kini-telah-operasikan-76-pesawat.

Ministry of Energy and Mineral Resources. 2016. "Data Inventory Emisi GRK Sektor Energi." Indonesia: Ministry of Energy and Mineral Resources.

Ministry of Energy and Mineral Resources. 2017. "Kajian Penggunaan Faktor Emisi Lokal (Tier 2) dalam Inventarisasi GRK Sektor Energi." Indonesia: Ministry of Energy and Mineral Resources. www.esdm.go.id/assets/media/content/ content-kajian-emisi-gas-rumah-kaca-2017.pdf.

Ministry of Energy and Mineral Resources. 2019. "Updated Emission Factor for Energy Sector." Pusat Data dan Informasi, Ministry of Energy and Mineral Resources

Ministry of Environment and Forestry. 2010. "Peraturan Menteri Negara Lingkungan Hidup Nomor 12 Tahun 2010-Hukumonline.Com." Ministry of Environment and Forestry Indonesia. https://m.hukumonline.com/pusatdata/ detail/tt4c283ee4c67c5/node/lt511a13750665b/peraturan-menteri-negaralingkungan-hidup-no-12-tahun-2010-pelaksanaan-pengendalian-pencemaranudara-di-daerah.

Ministry of Environment and Forestry. 2017. "Peraturan Menteri Lingkungan Hidup No 73 Tahun 2017 Tentang Pedoman Penyelenggaraan Dan Pelaporan Inventarisasi Gas Rumah Kaca Nasional." Ministry of Environment and Forestry Indonesia. http://jdih.menlhk.co.id/index.php/permenlhk/search.

Ministry of National Development Planning، 2020. "Indonesia Mid-Term Development Plan 2020-2024. Presidential Regulation No 18 2020".

Ministry of Transportation. 2014, "Land Transport in Figure." Indonesia Ministry of Transportation.
Ministry of Transportation. 2018. "Transportation Statistics 2018." http:// dephub.go.id/public/files/uploads/posts/ppidposts/postbody/bukustatistik-1-2018-final.pdf.

Noussan, Michel, and Simone Tagliapietra. 2020. "The Effect of Digitalization in the Energy Consumption of Passenger Transport: An Analysis of Future Scenarios for Europe." Journal of Cleaner Production 258 (June): 120926. https://doi.org/10,1016/i.jclepro.2020.120926.

Oanh, N. T. K., D. A. Permadi, N. P Dong, D. A. Nguyet. 2018. "Emission of Toxic Air Pollutants and Greenhouse Gases from Crop Residue Open Burning in Southeast Asia."

In Land-Atmospheric Research Applications in South and Southeast Asia, Springer Remote Sensing/Photogrammetry, edited by Krishna Prasad Vadrevu, Toshimasa Ohara, and Chris Justice, 47-66. Cham: Springer International Publishing.

Olmer, Naya, Bryan Comer, Biswajoy Roy, Xiaoli Mao, and Dan Rutherford. 2017. "Greenhouse Gas Emissions from Global Shipping, 2013-2015." International Council on Clean Transportation. https://theicct.org/ publications/GHG-emissions-global-shipping-2013-2015.

Ritchie, Hannah. 2020. "Cars, Planes, Trains: Where Do CO Emissions from Transport Come From?" Our World in Data. October 6. https:// ourworldindata.org/co2-emissions-from-transport.

Rizki, Muhamad, Dewi R. Sari, Nanda A. Noor, Imam Basuki, Rinaldi Imanuddin, Seny Damayanti, and Nadia Irwanto. 2020. "Indonesia Zero Emissions Application (EMISI): Methodologies for Calculating Urban Transport Emissions and Tree Sequestration." https://wri-indonesia.org/en/ publication/technical-note-emisi-app-urban-transport-tree.

Roberts, Mark, Frederico Gil Sander, and Sailesh Tiwari. 2019. "Augment, Connect, Target: Realizing Indonesia's Urban Potential." Text/HTML. World Bank. www.worldbank.org/en/country/indonesia/publication/augmentconnect-target-realizing-indonesias-urban-potential.

Shrestha, R. M., N. T. Kim Oanh, R. Shrestha, M. Rupakheti, D. A. Permadi, T. Kanabkaew, and R. Salony 2013. Atmospheric Brown Clouds Emission Inventory Manual. Nairobi, Kenya: United Nations Environmental Programme.

Sims, Ralph, Roberto Schaeffer, Felix Creutzing, Xochitl Cruz-Núñez, Marcio D’Agosto, Delia Dimitriu, Maria Josefina Figueroa Meza, et al. 2015. "Transport." Climate Change 2014: Mitigation of Climate Change.

Contribution of Working Group III to the Fifth Assessment Report of the Intergovernmental Panel on Climate Change. www.ipcc.ch/report/ar5/wg3/ transport/. 
Song, S. 2017. Transport Emissions \& Social Cost Assessment: Methodology Guide. Washington, DC: World Resources Institute. https://files.wri.org/s3fspublic/Transport Emissions Social Cost Assessment Methodology Guide.pdf.

tiki.id 2021. Lokasi Tiki. https://tiki.id/id/location.

Tokopedia. (2021). Berat dan Ukuran Paket Benar, Kunci Pengiriman Lancar. https://seller.tokopedia.com/edu/cara-menghitung-berat-paket/.

United Nations. 2020. 2017 Energy Balances. United Nations. https://doi. org/10.18356/c6edd9a4-en.

US EPA (US Environmental Protection Agency). 2021. "Inventory of U.S. Greenhouse Gas Emissions and Sinks: 1990-2019." Reports and Assessments. US EPA. February 3. www.epa.gov/ghgemissions/inventoryus-greenhouse-gas-emissions-and-sinks-1990-2019.

Utami, Tri Kusumaning. 2020. "Kajian Evaluasi Lintas Penyeberangan Merak-Bakauheni." Warta Penelitian Perhubungan 32 (1): 43-52. https://doi. org/10.25104/warlit.v32i1.1543.

Wang, Shiying, and Mengpin Ge. 2019. "Everything You Need to Know About the Fastest-Growing Source of Global Emissions: Transport." October. www. wri.org/insights/everything-you-need-know-about-fastest-growing-sourceglobal-emissions-transport. 


\section{ACKNOWLEDGMENTS}

We are pleased to acknowledge our various institutional strategic partners who provide core funding to the World Resources Institute (WRI). Their bilateral funding for projects made this publication possible.

The authors would like to thank the following people for providing invaluable insight and assistance in the development of this paper: Dean Affandi (WRI Indonesia), Emilia Suarez (WRI), Renee Pineda (WRI), and Gregory Taff (WRI).

We also thank our internal and external reviewers, Iris Chang (Grab), Tyas Widyastuti (Grab), Indira Darmoyono (Masyarakat Transportasi Indonesia), Didin Agustian Permadi (ITENAS), Xiang Li (WRI), Beatriz Cárdenaz (WRI), and Su Song (WRI).

The authors would also like to thank Sakinah Haniy, Achmad Noviansyah, Romain Warnault, Terra Komunika, Farhan Fahrezi, Septika Sihite, and Aulia Lastriarsi for their extensive support during the editing and design of this study. Opinions or points of view expressed in this report are those of the authors and do not necessarily reflect the position of the institution, reviewers, or our partners.

\section{ABOUT THE AUTHORS}

\section{Muhamad Rizki}

Mobility and Transport Specialist, WRI Indonesia

Lecturer, Civil Engineering Department,

Institut Teknologi Nasional Bandung

muhamad.rizki@wri.org

\section{Jeanly Syahputri}

Former Transportation Intern, WRI Indonesia

School of Transport Science, Hasselt University

jeanlysyahputri@gmail.com

\section{Nanda A. Noor}

Sustainable Landscape \& Business Senior Lead, WRI Indonesia nanda.noor@wri.org

\section{Philipe G. Gan}

Former Data Scientist Intern, WRI Indonesia PhD Candidate, Australian National University philipe.gunawan@anu.edu.au

\section{Retno Wihanesta}

Urban Transport Planner, WRI Indonesia retno.wihanesta@wri.org

\section{ABOUT WRI}

World Resources Institute is a global research organization that turns big ideas into action at the nexus of environment, economic opportunity, and human well-being.

\section{Our Challenge}

Natural resources are at the foundation of economic opportunity and human well-being. But today, we are depleting Earth's resources at rates that are not sustainable, endangering economies and people's lives. People depend on clean water, fertile land, healthy forests, and a stable climate. Livable cities and clean energy are essential for a sustainable planet. We must address these urgent, global challenges this decade.

\section{Our Vision}

We envision an equitable and prosperous planet driven by the wise management of natural resources. We aspire to create a world where the actions of government, business, and communities combine to eliminate poverty and sustain the natural environment for all people.

\section{Our Approach}

\section{COUNT IT}

We start with data. We conduct independent research and draw on the latest technology to develop new insights and recommendations. Our rigorous analysis identifies risks, unveils opportunities, and informs smart strategies. We focus our efforts on influential and emerging economies where the future of sustainability will be determined.

\section{CHANGE IT}

We use our research to influence government policies, business strategies, and civil society action. We test projects with communities, companies, and government agencies to build a strong evidence base. Then, we work with partners to deliver change on the ground that alleviates poverty and strengthens society. We hold ourselves accountable to ensure our outcomes will be bold and enduring.

\section{SCALE IT}

We don't think small. Once tested, we work with partners to adopt and expand our efforts regionally and globally. We engage with decision-makers to carry out our ideas and elevate our impact. We measure success through government and business actions that improve people's lives and sustain a healthy environment.

Maps are for illustrative purposes and do not imply the expression of any opinion on the part of WRI concerning the legal status of any country or territory or concerning the delimitation of frontiers or boundaries. 